Draft: November 6, 2018

\title{
Domain walls out of equilibrium
}

\author{
S.M. Alamoudi ${ }^{(a)}$ 円, D. Boyanovsky ${ }^{(a)}$ [य and F.I. Takakura ${ }^{(a, b)}$ ] \\ (a) Dept. of Physics and Astronomy, University of Pittsburgh, Pittsburgh PA USA 15260 \\ (b) Departamento de Fúsica, Instituto de Ciências Exatas, \\ Universidade Federal de Juiz de Fora, Juiz de Fora, 36036-330, MG, Brasil
}

\begin{abstract}
We study the non-equilibrium dynamics of domain walls in real time for $\phi^{4}$ and Sine Gordon models in $1+1$ dimensions in the dilute regime. The equation of motion for the collective coordinate is obtained by integrating out the meson excitations around the domain wall to oneloop order. The real-time non-equilibrium relaxation is studied analytically and numerically to this order. The constant friction coefficient vanishes but there is dynamical friction and relaxation caused by off-shell non-Markovian effects. The validity of a Markovian description is studied in detail. The proper Langevin equation is obtained to this order, the noise is Gaussian and additive but colored. We analyze the classical and hard thermal loop contributions to the self-energy and noise kernels and show that at temperatures larger than the meson mass the hard contributions are negligible and the finite temperature contribution to the dynamics is governed by the classical soft modes of the meson bath. The long time relaxational dynamics is completely dominated by classical Landau damping resulting in that the corresponding time scales are not set by the temperature but by the meson mass. The noise correlation function and the dissipative kernel obey a generalized form of the Fluctuation-Dissipation relation.
\end{abstract}

PACS: 11.90.+t; 71.45.-d; 72.15.Nj.

Keywords: Nonequilibrium; Quantum Fields.

\footnotetext{
${ }^{1}$ Email: smast15@vms.cis.pitt.edu

${ }^{2}$ Email: boyan@vms.cis.pitt.edu

${ }^{3}$ Email: takakura@fisica.ufjf.br
} 


\section{INTRODUCTION AND MOTIVATION}

Kinks and domain walls play a fundamental role in the equilibrium and non-equilibrium processes after phase transitions to broken symmetry states in theories with scalar order parameters. In scalar field theories that undergo a symmetry breaking phase transition the process of phase ordering proceeds by the formation of domains of the ordered phase separated by domain walls. These domain walls are topological defects that separate regions in which the order parameter is locally ordered and therefore locally the system is in a broken symmetry ground state in each domain. Interest in the dynamics of these topological excitations is interdisciplinary. In condensed matter systems solitons (or kinks) are collective excitations in quasi-one dimensional charge density wave systems and conducting polymers [1, 2, 3]. In particle physics domain walls in the form of sphalerons [4] have been argued to play an important role in baryogenesis[⿰], and in cosmology domain walls and other topological defects could be responsible for structure formation[6, 7].

The classical statistical mechanics of a gas of kinks in one spatial dimension has been previously studied[[8] and it was established that the kink density at a temperature $\mathrm{T}$ is approximately given by $n_{K} \approx e^{-M / T}$ with $M$ being the kink mass. Therefore a study of the dynamics of single domain walls or kinks will be valid in the dilute regime $M>T$ in which the interaction between kinks can be ignored because the mean separation between kinks is much larger than the typical width of a kink (of the order of the zero temperature correlation length or inverse meson mass).

The focus of this article is to study the real time dynamics of relaxation of domain walls (kinks) in $1+1$ dimensions via the interaction between the domain wall and the meson fluctuations in model field theories. In particular we study in detail scalar $\phi^{4}$ and Sine-Gordon kinks in the dilute regime in which $T<<M$. This problem is important in particle physics, condensed matter and cosmology. In particle physics dissipative processes on the dynamics of sphaleron configurations are important to establish corrections to the proper transition rates. In quasi-one dimensional condensed matter systems kinks and domain walls are responsible for important transport phenomena and therefore a study of the dissipative aspects will provide a deeper understanding of these phenomena. In cosmology the evolution of domain walls or dynamic of interfaces determines the scales in which ordering of horizon sized regions occur.

Although a study in $1+1$ dimension may not be a proper realization of the $3+1$ dimensional situations in particle physics and cosmology, it will at least highlight important aspects of the dynamics that must be generalized to the proper situations.

In condensed matter there is a considerable effort in understanding dissipative aspects of solitons starting from a microscopic description [9]-[13] in terms of Mori's formulation of linear response, and more recently in terms of a system-bath formulation 13.

Recently Khlebnikov 14 has studied the velocity of a bubble wall in the case of a non-degenerate scalar potentials. The bubble-wall velocity was related to the self-energy of the scalar field through the fluctuation-dissipation theorem. Assuming a trilinear coupling to another massive field a local 
friction coefficient was extracted. Alternatively, Arnold[15] provided an equivalent result to the one obtained in Ref. [14] at one loop using reflection and transmission coefficients for particles scattering off the bubble wall.

Our approach is rather different. It is tailored to obtain a real time description of the dissipative processes and a consistent derivation of the Langevin equation in a weak coupling perturbative expansion. The main ingredient is the collective coordinate quantization of the kink that allows to obtain the non-equilibrium generating functional for the collective coordinate by integrating out the meson degrees of freedom, i.e. the fluctuations around the kink. The resulting Langevin equation allows an unambiguous identification of the dissipative kernel and the noise correlation function that obey a generalized fluctuation dissipation relation. The dissipative processes arise from the interaction between the collective coordinate and the orthogonal fluctuations around the kink, rather than from the coupling to other fields.

We provide analytic and numerical study of the solutions of the equations of motion of the kink collective coordinate in lowest order (one loop) and establish that a Markovian approximation fails to describe the dynamics at large temperatures. Furthermore we analyze in detail the high temperature low density regime in which $m<<T<<M$ with $m$ being the meson mass focusing on the classical and hard thermal loop contribution to the dissipative kernel and the noise-noise correlation function. We argue that in lowest order in perturbation theory, the long time dynamics is completely dominated by classical Landau damping.

The main results of this article are the following

- A field-theoretical derivation of the real-time non-equilibrium equations of motion of the collective coordinate associated with translations of the domain wall and its solution in relevant cases for the sine-Gordon and $\phi^{4}$ potentials.

- A detailed microscopic derivation of the non-equilibrium influence functional, the quantum Langevin equation and the generalized fluctuation dissipation theorem to one-loop order.

- A detailed analytic and numerical study of the relaxation in the one-loop approximation. The Markovian approximation is compared to the "exact" dynamics in a wide range of temperature and the high temperature and classical limits analyzed in detail. The long time dynamics to this order is analyzed both analytically and numerically.

To our knowledge these aspects of domain wall dynamics had not been studied previously.

Section 2 summarizes briefly the main concepts in collective coordinate quantization that are relevant for our study. In section 3 we introduce the main tools of non-equilibrium field theory to study the kink in a bath of mesons in equilibrium and describe in general the relevant interactions, the equation of motion of the collective coordinate a Markovian approximation and the Langevin equation. Section 1 analyzes in detail the $\phi^{4}$ and Sine-Gordon models for which a Markovian approximation is shown to fail at large temperatures. In section 0 we study the high temperature 
but low density limit $(m<<T<<M)$ and establish that the long time dynamics is dominated by classical Landau damping processes. In section 6 we discuss higher order corrections to the results obtained within the one-loop approximation and we comment on generalization to higher dimensions. Section 7 presents our conclusions. Several appendices are included for technical details, in particular appendix 9 establishes the generalized fluctuation-dissipation relation between the damping kernel and the noise-noise correlation function.

\section{COLLECTIVE COORDINATE QUANTIZATION}

To begin our study of the dynamics of kinks we focus on $1+1$ dimensional quantum field theories described by Hamiltonians of the form

$$
H=\int d x\left\{\frac{\pi^{2}}{2}+\frac{1}{2}\left(\frac{d \phi}{d x}\right)^{2}+U(\phi)\right\}
$$

in which the potential $U(\phi)$ admits degenerate, broken symmetry minima.

A static kink is a solution of the time independent field equation

$$
-\frac{d^{2} \phi_{s}}{d x^{2}}+\frac{\partial U\left(\phi_{s}\right)}{\partial \phi}=0
$$

with boundary conditions such that $\phi_{s}(x \rightarrow \pm \infty)=\phi_{ \pm \infty}$ and $U\left(\phi_{ \pm \infty}\right)=0$ [16]-26]. Translational invariance implies that such solution is of the form $\phi_{s}\left(x-x_{0}\right)$ with $x_{0}$ an arbitrary translation chosen such that $\phi_{s}(0)=0$, therefore $x_{0}$ is identified with the position of the kink.

Lorentz invariance results in that a kink moving with constant velocity is given by $\phi_{s}\left[\frac{x-x_{0}-v t}{\sqrt{1-v^{2}}}\right]$ [16][19. The mass of the kink, i.e, the energy of a static kink is given by

$$
M \equiv E\left[\phi_{s}\right]=\int \mathrm{d} x\left(\frac{\mathrm{d} \phi_{s}}{\mathrm{~d} x}\right)^{2} .
$$

Quantization around the static kink solution implies writing

$$
\hat{\phi}(x, t)=\phi_{s}\left(x-x_{0}\right)+\hat{\psi}\left(x-x_{0} ; t\right) .
$$

Where the fluctuation operator is expanded in terms of a complete set of harmonic modes around the kink

$$
\hat{\psi}\left(x-x_{0} ; t\right)=\sum_{n}^{\infty} q_{n}(t) \mathcal{U}_{n}\left(x-x_{0}\right)
$$


where the mode functions $\mathcal{U}_{n}\left(x-x_{0}\right)$ obey

$$
\left[-\frac{\mathrm{d}^{2}}{\mathrm{~d} x^{2}}+\left.\frac{d^{2} U}{d \phi^{2}}\right|_{\phi_{s}}\right] \mathcal{U}_{n}\left(x-x_{0}\right)=\omega_{n}^{2} \mathcal{U}_{n}\left(x-x_{0}\right)
$$

with the completeness relation given by

$$
\sum_{\mathrm{b}} \mathcal{U}_{b}^{*}\left(x-x_{0}\right) \mathcal{U}_{b}\left(x^{\prime}-x_{0}\right)+\int d k \mathcal{U}_{k}^{*}\left(x-x_{0}\right) \mathcal{U}_{k}\left(x^{\prime}-x_{0}\right)=\delta\left(x-x^{\prime}\right)
$$

and the subscript $\mathrm{b}$ stands for summation over bound states and $\mathrm{k}$ for scattering states. For bound states, the eigenvectors are chosen to be real and for scattering states, we label them as $\mathcal{U}_{k}\left(x-x_{0}\right)$ and are chosen such that $\mathcal{U}_{k}^{*}=\mathcal{U}_{-k}$, in which case the coordinate operators obey the hermiticity condition $q_{k}^{*}(t)=q_{-k}(t)$.

These eigenvectors are normalized as

$$
\int \mathrm{d} x \mathcal{U}_{p}^{*}\left(x-\hat{x}_{0}\right) \mathcal{U}_{q}\left(x-\hat{x}_{0}\right)=\delta_{p, q}
$$

As a consequence of translational invariance, there is a mode with zero eigenvalue given by [16]19

$$
\mathcal{U}_{0}\left(x-x_{0}\right)=\frac{1}{\sqrt{M}}\left(\frac{\mathrm{d} \phi_{s}}{\mathrm{~d} x}\right)
$$

Depending on the particular form of the potential $U(\phi)$ there may be other bound states (as is the case with the $\phi^{4}$ potential). There is a continuum of scattering states with frequencies $\omega_{k}^{2}=$ $k^{2}+\omega_{o}^{2} ; \omega_{o}^{2}=d^{2} U(\phi) /\left.d^{2} \phi\right|_{\phi_{\infty}}$. These scattering states correspond asymptotically to phase shifted plane waves in the cases under consideration because the relevant potentials are reflectionless [16, 17]. The continuum states are identified with meson states, whereas bound states (other than the zero mode) are identified with excited states of the kink 18].

The fluctuation along the functional direction corresponding to the zero frequency mode represents an infinitesimal translation of the kink that costs no energy. Since this mode has no restoring force, any arbitrarily large amplitude fluctuation along this direction is energetically allowed and therefore must be treated non-perturbatively. The variable $x_{0}$, i.e. the center of mass of the kink is elevated to the status of a quantum mechanical variable, and the fluctuations are orthogonal to the zero mode. This treatment is the basis of the collective coordinate method [16, 19, 20]-[28].

In collective coordinates quantization instead of the expansion (4) with (5) we expand $\phi(x, t)$ as

$$
\phi(x, t)=\phi_{s}\left(x-\hat{x}_{0}(t)\right)+\sum_{n \neq 0}^{\infty} Q_{n}(t) \mathcal{U}_{n}\left(x-\hat{x}_{0}(t)\right) .
$$


This amounts to a change of basis in functional space, from the "cartesian" coordinates $\left\{q_{n}\right\}$ to "curvilinear" coordinates $\left\{\hat{x}_{0}, Q_{n \neq 0}\right\}$ 16, 19, 22, 27].

The next step is to express the Hamiltonian in terms of the new variables $\hat{x}_{0}(t)$ and $Q_{n}(t)$. For this we follow references 16, 19, 22, 27 and which we summarize below for the cases under consideration.

\subsection{Kinetic and potential energies}

In the Schroedinger representation the kinetic energy can be expressed as a functional derivative as

$$
T=-\frac{1}{2} \int \mathrm{d} x \frac{\delta}{\delta \phi} \frac{\delta}{\delta \phi}
$$

where the functional derivative is written in the new coordinates using the chain-rule

$$
\frac{\delta}{\delta \phi(x)}=\frac{\delta \hat{x}_{0}}{\delta \phi(x)} \frac{\delta}{\delta \hat{x}_{0}}+\sum_{m \neq 0} \frac{\delta Q_{m}}{\delta \phi(x)} \frac{\delta}{\delta Q_{m}} .
$$

Taking the functional variation of the field $\phi$, eq.(10), we obtain

$$
\begin{aligned}
\delta \phi(x) & =\frac{\delta \phi(x)}{\delta \hat{x}_{0}} \delta \hat{x}_{0}+\sum_{m \neq 0} \frac{\delta \phi(x)}{\delta Q_{m}} \delta Q_{m} \\
& =\left[\frac{\partial \phi_{s}\left(x-\hat{x}_{0}\right)}{\partial \hat{x}_{0}}+\sum_{m \neq 0} Q_{m} \frac{\partial \mathcal{U}_{m}\left(x-\hat{x}_{0}\right)}{\partial \hat{x}_{0}}\right] \delta \hat{x}_{0}+\sum_{n \neq 0} \mathcal{U}_{n}\left(x-\hat{x}_{0}\right) \delta Q_{n} .
\end{aligned}
$$

Projecting both sides of the above equation on $\mathcal{U}_{0}^{*}\left(x-\hat{x}_{0}\right)$ and then $\mathcal{U}_{p}^{*}\left(x-\hat{x}_{0}\right)$ with $p \neq 0$, using eqn.(9) and the orthonormalization condition eqn.(8), we obtain:

$$
\begin{aligned}
\frac{\delta \hat{x}_{0}}{\delta \phi(x)} & =-\frac{1}{\sqrt{M}} \frac{1}{\left[1+(1 / \sqrt{M}) \sum_{m \neq 0} Q_{m} S_{m}\right]} \mathcal{U}_{0}^{*}\left(x-\hat{x}_{0}\right) \\
\frac{\delta Q_{p}}{\delta \phi(x)} & =\mathcal{U}_{p}^{*}\left(x-\hat{x}_{0}\right)-\frac{1}{\sqrt{M}} \frac{\sum_{n \neq 0} G_{p n} Q_{n}}{\left[1+(1 / \sqrt{M}) \sum_{m \neq 0} Q_{m} S_{m}\right]} \mathcal{U}_{0}^{*}\left(x-\hat{x}_{0}\right),
\end{aligned}
$$

where the matrix elements $G_{p m}$ are defined as

$$
\begin{gathered}
G_{p m}=\int \mathrm{d} x \mathcal{U}_{p}^{*}\left(x-\hat{x}_{0}\right) \frac{\partial \mathcal{U}_{m}\left(x-\hat{x}_{0}\right)}{\partial x} \\
S_{m} \equiv G_{0 m}=\int \mathrm{d} x \mathcal{U}_{0}\left(x-\hat{x}_{0}\right) \frac{\partial \mathcal{U}_{m}\left(x-\hat{x}_{0}\right)}{\partial x} .
\end{gathered}
$$


At this stage it is straightforward to follow the procedure detailed in [19, 22, 27] to find the final form of the kinetic term in the Hamiltonian in the Schroedinger representation of the coordinates $\hat{x}_{0}, Q_{m \neq 0}$ :

$$
\begin{gathered}
T=-\frac{1}{2}\left\{\frac{1}{D} \frac{\delta}{\delta \hat{x}_{0}} \frac{\delta}{\delta \hat{x}_{0}}+\frac{1}{\sqrt{D}} \frac{\delta}{\delta \hat{x}_{0}} \sum_{p, m \neq 0}\left[\frac{G_{p m} Q_{m}}{\sqrt{D}} \frac{\delta}{\delta Q_{p}}+\frac{\delta}{\delta Q_{p}} \frac{G_{p m} Q_{m}}{\sqrt{D}}\right]+\right. \\
\left.\frac{1}{\sqrt{D}} \sum_{p, q, m, n \neq 0} \frac{\delta}{\delta Q_{p}}\left[\delta_{-p, q} \sqrt{D}+\frac{G_{p m} Q_{m}}{\sqrt{D}} G_{q n} Q_{n}\right] \frac{\delta}{\delta Q_{q}}\right\}
\end{gathered}
$$

where $\sqrt{D}$ is the Jacobian associated with the change of coordinates 16, 19, 22, 27] and given by

$$
\sqrt{D} \equiv \sqrt{M}\left[1+\frac{1}{\sqrt{M}} \sum_{m \neq 0} Q_{m} S_{m}\right]
$$

The total potential energy, including the elastic term, $V[\phi]$ (see eqn.(1)), is given by

$$
V[\phi] \equiv \int \mathrm{d} x\left[\frac{1}{2}\left(\frac{\partial \phi}{\partial x}\right)^{2}+U(\phi)\right] .
$$

Using the expansion given by eqn.(10) we find that it can be written in terms of the new coordinates as

$$
V[\phi]=M+\frac{1}{2} \sum_{m \neq 0} Q_{m} Q_{-m} \omega_{m}^{2}+\mathcal{O}\left(Q^{3}\right)+\cdots .
$$

By translational invariance the potential energy does not depend on the collective coordinate. Identifying the canonical momenta conjugate to $\hat{x}_{0}, Q_{n}$ as

$$
\pi_{0} \equiv P=-i \frac{\delta}{\delta \hat{x}_{0}} \quad ; \quad \pi_{k}=-i \frac{\delta}{\delta Q_{-k}} \quad \text { for } \quad k \neq 0
$$

and using the commutation relation of $\sqrt{D}$ and $1 / \sqrt{D}$ with $Q_{n}, \pi_{n}$ and $P$ given by

$$
\left[\pi_{n}, \sqrt{D}\right]=-i S_{n} \quad \text { and } \quad ; \quad\left[\pi_{n}, \frac{1}{\sqrt{D}}\right]=-i \frac{S_{n}}{D}
$$

we find the final form of the Hamiltonian: 


$$
\begin{aligned}
H=M+ & \frac{1}{2}\left\{\frac{P^{2}}{D}+\frac{P}{\sqrt{D}} \sum_{p, m \neq 0}\left[\frac{G_{p m} Q_{m}}{\sqrt{D}} \pi_{-p}+\pi_{-p} \frac{G_{p m} Q_{m}}{\sqrt{D}}\right]+\sum_{p \neq 0} \omega_{p}^{2} Q_{p} Q_{-p}+\right. \\
& \left.\frac{1}{\sqrt{D}} \sum_{p, q, m, n \neq 0} \pi_{-p}\left[\delta_{-p, q} \sqrt{D}+\frac{G_{p m} Q_{m}}{\sqrt{D}} G_{q n} Q_{n}\right] \pi_{-q}\right\}+\mathcal{O}\left(Q^{3}\right)+\cdots,
\end{aligned}
$$

where $Q_{p}$ are now operators. The coordinates $Q_{k}$ associated with the scattering states describe the meson degrees of freedom with frequency $\omega^{2}(k)=k^{2}+\omega_{o}^{2} ; \omega_{o}^{2}=d^{2} U(\phi) /\left.d^{2} \phi\right|_{\phi_{\infty}}$. Since the Hamiltonian does not depend on $\hat{x}_{0}$ its canonical momentum $P$ is conserved, it is identified with the total momentum of the kink-meson system[16, 19, 22]. The kink velocity, however, is not proportional to $P$ and depends on the momentum of the meson field.

Since our goal is to study the dynamics of the kink by obtaining the equation of motion for the expectation value of the kink collective coordinate, we introduce an external source term linearly coupled to $\hat{x}_{0}$. This source term has a dual purpose, one is to allow to obtain the correlation function of the collective coordinate as functional derivatives with respect to this source, the other is to use this source as a Lagrange multiplier to turn the evolution equation into an initial value problem. This second use will become clear later when we study the solutions to the equations of motion. Therefore we add the term $j(t) \hat{x}_{0}$ to the Hamiltonian.

\section{A DOMAIN WALL IN THE MESON HEAT BATH}

Our goal is to study the dynamics of a domain wall in interaction with the mesons. This is achieved by obtaining the real-time equations of motion of the collective coordinate $\hat{x}_{0}$ by treating the mesons as a "bath" and obtaining an influence functional[29]-34] by "tracing out" the meson degrees of freedom and the excited states of the kink. We assume that the total density matrix for the kink-meson system decouples at the initial time $t_{i}$, i.e.

$$
\rho\left(t_{i}\right)=\rho_{s}\left(t_{i}\right) \otimes \rho_{R}\left(t_{i}\right),
$$

where $\rho_{s}\left(t_{i}\right)$ is the density matrix of the system which is taken to be that of a free particle associated with the collective coordinate of the kink, i.e. $\rho_{s}\left(t_{i}\right)=\left|x_{0}><x_{0}\right|$ and $\rho_{R}\left(t_{i}\right)$ is the density matrix of the meson bath and describes mesons in thermal equilibrium at a temperature $T$.

Since the kinks can never be separated from the meson fluctuations, this factorization must be understood to hold in the limit in which the initial time $t_{i} \rightarrow-\infty$ with an adiabatic switching of the kink-meson interaction.

The time evolution is completely contained in the time dependent density matrix

$$
\rho(t)=U\left(t, t_{i}\right) \rho\left(t_{i}\right) U^{-1}\left(t, t_{i}\right)
$$


with $U\left(t, t_{i}\right)$ the time evolution operator. Real time non-equilibrium expectation values and correlation functions can be obtained via functional derivatives with respect to sources of the generating functional[35]-42]

$$
Z\left[j^{+}, j^{-}\right]=\operatorname{Tr} U\left(\infty,-\infty ; j^{+}\right) \rho_{i} U^{-1}\left(\infty,-\infty ; j^{-}\right) / \operatorname{Tr} \rho\left(t_{i}\right),
$$

where $j^{ \pm}$are sources coupled to the meson field and the collective coordinate. This generating functional is readily obtained using the Schwinger-Keldysh method which involves a path integral in a complex contour in time 35]-43]: a branch corresponding to the time evolution forward, a backward branch corresponding to the inverse time evolution operator and a branch along the imaginary time axis from $t_{i}$ to $t_{i}-i \beta$ to represent the initial thermal density matrix. We will obtain the equation of motion for the kink collective coordinate in an expansion of the "adiabatic" parameter $\omega_{0} / M$ which is also the weak coupling limit of the scalar field theories under consideration [16]. As it will be shown explicitly below in the particular cases studied, the matrix elements given by eqns.(16,17) will provide the necessary powers of the meson mass $\omega_{0}$. The lowest order in $\omega_{0} / M$ is formally obtained by keeping only the $1 / M$ terms in the Hamiltonian and neglecting the non-linear $\mathcal{O}\left(Q^{3}\right)$ terms. Under these approximations, $1 / D \simeq 1 / M$ and the Hamiltonian has the following form

$$
H=M+\frac{1}{2 M}\left(P+\sum_{m, n \neq 0} D_{m n} \pi_{m} Q_{n}\right)^{2}+\frac{1}{2} \sum_{m \neq 0}\left[\pi_{m} \pi_{-m}+\omega_{m}^{2} Q_{m} Q_{-m}\right]+j(t) \hat{x}_{0},
$$

where we define

$$
D_{m n}=G_{-m n}
$$

At this point it proves convenient to write the coordinates and momenta of the mesons in terms of creation and annihilation operators obeying the standard Bose commutation relations,

$$
Q_{k}=\frac{1}{\sqrt{2 \omega_{k}}}\left[a_{k}+a_{-k}^{\dagger}\right] ; \pi_{k}=-i \sqrt{\frac{\omega_{k}}{2}}\left[a_{k}-a_{-k}^{\dagger}\right] \text {. }
$$

The Hamiltonian can be expressed in terms of $a$ and $a^{\dagger}$ as

$$
H=\frac{1}{2 M}\left(P+F\left[a^{\dagger}, a\right]\right)^{2}+\sum_{k \neq 0} \omega_{k}\left(a_{k}^{\dagger} a_{k}+1 / 2\right)+j(t) \hat{x}_{0}+M,
$$

where

$$
F\left[a^{\dagger}, a\right]=\sum_{p, k \neq 0}\left[T_{p k}^{(S)}\left(a_{p} a_{k}-a_{-p}^{\dagger} a_{-k}^{\dagger}\right)+T_{p k}^{(A)}\left(a_{-p}^{\dagger} a_{k}-a_{-k}^{\dagger} a_{p}\right)\right]
$$

We have made use of the symmetries of the operators and defined the symmetric $T_{p k}^{(S)}$ and antisymmetric $T_{p k}^{(A)}$ matrices that provide the interaction vertices as

$$
T_{k p}^{(S)}=\frac{1}{4 i}\left[\sqrt{\frac{\omega_{k}}{\omega_{p}}}-\sqrt{\frac{\omega_{p}}{\omega_{k}}}\right] D_{k p} \quad ; \quad T_{k p}^{(A)}=\frac{1}{4 i}\left[\sqrt{\frac{\omega_{k}}{\omega_{p}}}+\sqrt{\frac{\omega_{p}}{\omega_{k}}}\right] D_{k p} .
$$


To use the path integral formulation we need the Lagrangian, which to the order that we are working $\left(\mathcal{O}\left(\omega_{0} / M\right)\right)$ and properly accounting for normal ordering, is given by

$$
\mathcal{L}\left[\dot{\hat{x}_{0}}, a, a^{\dagger}\right]=\frac{M}{2}{\dot{\hat{x}_{0}}}^{2}-\dot{\hat{x}}_{0} F\left[a^{\dagger}, a\right]-\sum_{k \neq 0} \omega_{k}\left(a_{k}^{\dagger} a_{k}+1 / 2\right)-j(t) \hat{x}_{0}-M .
$$

The interaction of the collective coordinate and the mesons is now clear. Only time derivatives of the collective coordinate couple, a consequence of the Goldstone character of the collective coordinate. There are two processes described by the interaction: i) creation and destruction of two mesons and ii) scattering of mesons. Whereas the first type can contribute with the mesons in their ground state, the second can only contribute if the meson states are occupied. The two processes are depicted in fig.(C). As it will become clear below, the second type of processes will lead to Landau damping.

Since we have preferred to work in terms of the creation and annihilation operators it is convenient to write the path integral for the non-equilibrium generating functional in the coherent state representation [13, 44].

Following the steps outlined in reference 13, 44 we find the generating functional of nonequilibrium Green's functions in the coherent state representation to be given by

$\mathcal{Z}\left[j^{+}, j^{-}\right]=\int \mathcal{D} x^{+} \int \mathcal{D} x^{-} \int \mathcal{D}^{2} \gamma^{+} \int \mathcal{D}^{2} \gamma^{-} \exp \left\{i \int d t\left(\mathcal{L}\left[\dot{x}^{+}, \gamma^{*+}, \gamma^{+}, j^{+}\right]-\mathcal{L}\left[\dot{x}^{-}, \gamma^{-}, \gamma^{*-}, j^{-}\right]\right)\right\}$

with the Lagrangian density defined on each branch given by

$$
\begin{aligned}
\mathcal{L}\left[\dot{x}^{ \pm}, \gamma^{ \pm}, \gamma^{* \pm}, j^{ \pm}\right]= & \frac{M}{2}\left(\dot{x}^{ \pm}\right)^{2}+\sum_{k \neq 0}\left[i \gamma_{k}^{* \pm} \frac{d \gamma_{k}^{ \pm}}{d t}-\omega_{k} \gamma_{k}^{* \pm} \gamma_{k}^{ \pm}+\gamma_{k}^{ \pm} j_{k}^{* \pm}+\gamma_{k}^{* \pm} j_{k}^{ \pm}\right]- \\
& \dot{x}^{ \pm} F\left[\gamma^{* \pm}, \gamma^{ \pm}\right]-j(t) x^{ \pm}
\end{aligned}
$$

and with proper boundary conditions on the fields that reflect the factorized initial condition with the mesons in thermal equilibrium. The signs \pm in the above expressions correspond to the fields and sources on the forward $(+)$ and backward $(-)$ branches. The contribution from the branch along the imaginary time is cancelled by the normalization factor. This is the non-equilibrium generalization of the coherent state path integrals (see details in appendix A). Non-equilibrium Green's functions are now obtained as functional derivatives with respect to the sources $j^{ \pm}$. There are 4 types of free meson propagators 35- 42 :

$$
\begin{aligned}
<a_{k}^{\dagger+}(t) a_{p}^{+}\left(t^{\prime}\right)> & =\delta_{k, p} e^{-i \omega_{k}\left(t^{\prime}-t\right)}\left[\theta\left(t^{\prime}-t\right)+n_{k}\right] \\
<a_{k}^{+}(\tau) a_{p}^{\dagger+}\left(\tau^{\prime}\right)> & =\delta_{k, p} e^{i \omega_{k}\left(t^{\prime}-t\right)}\left[\theta\left(t-t^{\prime}\right)+n_{k}\right] \\
<a_{k}^{\dagger( \pm)}(t) a_{p}^{\dagger( \pm)}\left(t^{\prime}\right)> & =0
\end{aligned}
$$




$$
\begin{aligned}
<a_{k}^{( \pm)}(t) a_{p}^{( \pm)}\left(t^{\prime}\right)> & =0 \\
<a_{k}^{\dagger+}(\tau) a_{p}^{-}\left(\tau^{\prime}\right)> & =\delta_{k, p} e^{-i \omega_{k}\left(t^{\prime}-t\right)}\left[1+n_{k}\right] \\
<a_{k}^{+}(\tau) a_{p}^{\dagger-}\left(\tau^{\prime}\right)> & =\delta_{k, p} e^{i \omega_{k}\left(t^{\prime}-t\right)} n_{k},
\end{aligned}
$$

where $n_{k}$ is Bose Einstein distribution for mesons of quantum number $k$ and $\langle\cdots\rangle$ refer to averages in the initial density matrix. The $++(--)$ propagators correspond to the time-ordered (anti-time-ordered), whereas the $\pm \mp$ are the Wightman functions.

An important point to notice is that

$$
<F\left[a^{\dagger}, a\right]>=0
$$

in the non-interacting case, since it is proportional to $\sum_{k} D_{k,-k}=0$.

\subsection{The equation of motion for the collective coordinate}

The equation of motion of the expectation value of the collective coordinate for the kink $<\hat{x}_{0}>=$ $q(t)$, can be derived by expanding $x^{ \pm}(t)=q(t)+\xi^{ \pm}(t)$ and requiring $\left\langle\xi^{ \pm}(t)>=0\right.$ to all orders in perturbation theory 46. Imposing the condition $\left\langle\xi^{+}\left(\tau^{\prime}\right)\right\rangle=0$, treating the interaction term up to second order in perturbation theory and using eqn.(38), we obtain the following linearized equation of motion

$$
\int_{-\infty}^{\infty} d t^{\prime}<\xi^{+}(t) \dot{\xi}^{+}\left(t^{\prime}\right)>\left[\left\{M \dot{q}\left(t^{\prime}\right)+\int_{-\infty}^{t} d t^{\prime \prime} \Gamma_{m}\left(t^{\prime}-t^{\prime \prime}\right) \dot{q}\left(t^{\prime \prime}\right)\right\}+<\xi^{+}(t) \xi^{+}\left(t^{\prime}\right)>j\left(t^{\prime}\right)\right]=0
$$

where the retarded kernel is given by

$$
\begin{aligned}
-i \Gamma_{m}\left(t-t^{\prime}\right) \theta\left(t-t^{\prime}\right) & =<F\left[a^{\dagger+}(t), a^{+}(t)\right] F\left[a^{\dagger+}\left(t^{\prime}\right), a^{+}\left(t^{\prime}\right)\right]> \\
& -<F\left[a^{\dagger+}(t), a^{+}(t)\right] F\left[a^{\dagger-}\left(t^{\prime}\right) \cdot a^{-}\left(t^{\prime}\right)\right]>
\end{aligned}
$$

Since we restrict ourselves to non-relativistic kinks we consider $\dot{q} \ll 1$. The non-equilibrium Feynman diagrams that contribute to one loop order (second order) are shown in fig.(C).

Alternatively this equation of motion may be obtained by computing the influence functional[29][34] in second order perturbation theory. The resulting influence functional is quadratic in the collective coordinate, performing the shift $x^{ \pm}(t)=q(t)+\xi^{ \pm}(t)$ the above equation of motion is obtained by requesting that the linear terms in $\xi^{ \pm}$vanish, (there are two linear terms, both give the same equation of motion).

The kernel $\Gamma_{m}\left(t-t^{\prime}\right)$ is found by using eqn.(40) and eqn.(38) and it is given by

$$
\begin{aligned}
\Gamma_{m}\left(t-t^{\prime}\right)= & -4 \sum_{p, k \neq 0}\left\{T_{p k}^{(S)} T_{-p-k}^{(S)}\left(1+2 n_{p}\right) \sin \left[\left(\omega_{p}+\omega_{k}\right)\left(t-t^{\prime}\right)\right]\right. \\
& \left.-2 T_{p k}^{(A)} T_{-p-k}^{(A)} n_{p} \sin \left[\left(\omega_{p}-\omega_{k}\right)\left(t-t^{\prime}\right)\right]\right\} .
\end{aligned}
$$


Performing the integral over $t^{\prime}$ in eqn.(39) by parts, we obtain the final form of the equation of motion

$$
M \ddot{q}(t)+\int_{-\infty}^{t} \mathrm{~d} t^{\prime} \Sigma_{m}\left(t-t^{\prime}\right) \dot{q}\left(t^{\prime}\right)=j(t),
$$

where the non-local kernel is given by

$$
\Sigma_{m}\left(t-t^{\prime}\right)=\frac{\partial \Gamma_{m}\left(t-t^{\prime}\right)}{\partial t}=-\frac{\partial \Gamma_{m}\left(t-t^{\prime}\right)}{\partial t^{\prime}}
$$

Using eqn.(41) we find the final expression for the kernel $\Sigma_{m}$ :

$$
\begin{aligned}
\Sigma_{m}\left(t-t^{\prime}\right)= & -4 \sum_{p, k \neq 0}\left\{T_{p k}^{(S)} T_{-p-k}^{(S)}\left(1+2 n_{p}\right)\left(\omega_{p}+\omega_{k}\right) \cos \left[\left(\omega_{p}+\omega_{k}\right)\left(t-t^{\prime}\right)\right]\right. \\
& \left.-2 T_{p k}^{(A)} T_{-p-k}^{(A)} n_{p}\left(\omega_{p}-\omega_{k}\right) \cos \left[\left(\omega_{p}-\omega_{k}\right)\left(t-t^{\prime}\right)\right]\right\} .
\end{aligned}
$$

We will see in the next sections that the two kernels $\Sigma_{m} ; \Gamma_{m}$ have very special significance: whereas $\Sigma_{m}$ is identified with the real-time retarded self-energy of the collective coordinate, $\Gamma_{m}$ will provide the coefficient of dynamical friction in the Markovian approximation.

It is more convenient to express the equation of motion of the kink in terms of the velocity

$$
M \dot{V}(t)+\int_{-\infty}^{t} \mathrm{~d} t^{\prime} \Sigma_{m}\left(t-t^{\prime}\right) V\left(t^{\prime}\right)=j(t)
$$

with $\Sigma_{m}$ given by eqn.(44).

The relation (44), ensures to this order in the perturbative expansion, that with an adiabatic switching on convergence factor introduced to regularize the lower limit of the integral and to provide an initial factorization of the density matrix as $t_{i} \rightarrow-\infty$ the total integral of the retarded self-energy kernel vanishes, i.e,

$$
\int_{-\infty}^{t} \Sigma_{m}\left(t-t^{\prime}\right) d t^{\prime}=0
$$

This result is consistent with that found in Refs. [14, 15.

Therefore for $j=0$, any constant velocity of the kink is a solution of the equation of motion (45). This result is physically clear: when $j=0$, the total Hamiltonian commutes with $P$, the canonical momentum conjugate to $\hat{x}_{0}$ because of translational invariance, i.e. the total momentum of the system is conserved. One can then go to a frame in which $P=0$ and since the meson bath is in equilibrium this must result in that the domain wall must have a constant velocity, therefore for $j=0$ there must be a constant velocity solution to the equations of motion of the collective coordinate resulting in (46). 


\subsection{General properties of the solution}

Since in the absence of an external driving term we have found that the domain wall moves with constant velocity, we can now use the external source term to cast the evolution as an initial value problem. For this consider the situation in which at time $t=0$ a force is applied, therefore changing the velocity of the domain wall. Assuming that for $t<0$ the kink traveled with a constant velocity $v_{0}$, after switching on the external force the domain wall will accelerate, but it will also transfer energy and excite the meson degrees of freedom and this will lead to dissipative processes. Therefore writing $V(t)=v_{0}+v(t)$ with $j(t<0)=0 ; j(t>0) \neq 0$ and using the property (46) the equation of motion for the velocity change becomes

$$
M \dot{v}(t)+\int_{0}^{t} \mathrm{~d} t^{\prime} \Sigma_{m}\left(t-t^{\prime}\right) v\left(t^{\prime}\right)=j(t)
$$

The solution of this equation is found by Laplace transform, in terms of $\tilde{v}(s) ; \tilde{\Sigma}_{m}(s) ; \tilde{J}(s)$, the Laplace transforms of the velocity, self-energy kernel and current respectively, in terms of the Laplace variable $s$. We find that the solution is given by

$$
\tilde{v}(s)=\frac{v_{0}+(\tilde{J}(s) / M)}{s+\frac{1}{M} \tilde{\Sigma}_{m}(s)},
$$

and consistently with the linearized equation of motion and the non-relativistic dynamics $v(t) ; v_{0} \ll$ 1. The quantity

$$
G(s)=\frac{1}{s+\frac{1}{M} \tilde{\Sigma}_{m}(s)}
$$

is the Laplace transform of the propagator of the velocity of the collective coordinate. The real time evolution is found by the inverse Laplace transform

$$
v(t)=\frac{1}{2 \pi i} \int_{C} e^{s t} \tilde{v}(s) d s
$$

where $C$ refers to the Bromwich contour running along the imaginary axis to the right of all the singularities of $\tilde{v}(s)$ in the complex $s$ plane. Therefore we need to understand the analytic structure of $G(s)$ in eqn. (48) to obtain the real time dynamics. The Laplace transform of the self-energy kernel is conveniently written as a dispersion relation in the form

$$
\begin{aligned}
\tilde{\Sigma}_{m}(s)= & s \tilde{\Gamma}_{m}(s) \\
\tilde{\Gamma}_{m}(s)= & \int \frac{\rho\left(p_{o}\right)}{s^{2}+p_{o}^{2}} d p_{o} \\
\rho\left(p_{o}\right)= & -4 \sum_{p, k \neq 0}\left\{T_{p k}^{(S)} T_{-p-k}^{(S)}\left(1+2 n_{p}\right) \delta\left(p_{o}-\omega_{p}-\omega_{k}\right)\right. \\
& \left.-T_{p k}^{(A)} T_{-p-k}^{(A)}\left(n_{p}-n_{k}\right) \delta\left(p_{o}-\omega_{p}+\omega_{k}\right)\right\},
\end{aligned}
$$


where $\tilde{\Gamma}_{m}(s)$ is the Laplace transform of the kernel $\tilde{\Gamma}_{m}$ given above.

This dispersive form for the Laplace transform of the kernel reveals that $\tilde{\Gamma}_{m}(s)$ has a discontinuity in the complex s-plane along the imaginary axis, since

$$
\tilde{\Gamma}_{m I}\left(s=i \omega+0^{ \pm}\right)=\mp \frac{\pi \operatorname{sign}(\omega)}{2|\omega|}[\rho(|\omega|)-\rho(-|\omega|)]
$$

The imaginary part changes sign with $\omega$ as a result of the retarded nature of the kernel. Therefore the propagator $G(s)$ has cuts along the imaginary axis in the complex s-plane. The two different contributions to the spectral density (53) yield to two different cut structures. For $\omega>0$, the first term, proportional to $\delta\left(\omega-\omega_{p}-\omega_{k}\right)$ gives a two-meson cut beginning at $2 \omega_{0}$ corresponding to the process of spontaneous and induced two-meson creation and annihilation. The second contribution corresponding to $\delta\left(\omega-\omega_{p}+\omega_{k}\right)$ gives a cut, which we identify as the Landau damping cut 47, 46], pinching the origin and originates in the process of scattering of mesons present in the medium off the domain wall. As it will be seen in detail for the examples in the next section the discontinuity vanishes linearly as $\omega \rightarrow 0$ allowing an analytic continuation into the second Riemann sheet and to isolate the pole. This linear vanishing of the self energy is consistent with the case studied by [14, 15]. This process is present only for finite temperature as there must be mesons present for this process to exist. This contribution is identified as Landau damping from the in medium mesons and will be seen to provide the leading contribution to the long time relaxation.

The presence of a static friction coefficient will be revealed by a pole in $G(s)$ with a negative real part, since this will translate into an exponential relaxation of the velocity.

In the absence of interactions $G(s)$ has a simple pole at $s=0$. Since we obtained the expression for the kernels in perturbation theory the position of a pole must be found in a consistent perturbative expansion by writing $s_{p}=(1 / M) s_{1}+\cdots$, we find

$$
s_{p}=-\frac{1}{M} \tilde{\Sigma}_{m}(s=0) \equiv 0 .
$$

Therefore the coefficient of static friction vanishes. This is a consequence of the vanishing of the integral (46). Therefore up to this order in perturbation theory the position of the pole in the s-variable remains at $s=0$ resulting in that the static friction coefficient vanishes.

In summary, the analytic structure of $G(s)$ in the complex s-plane corresponds to a pole at $s=0$ with residue

$$
Z_{s}=\frac{1}{1+\frac{1}{M} \tilde{\Gamma}_{m}(0)}
$$

and cuts along the imaginary axis beginning at $\pm 2 i \omega_{o} ; \pm i \epsilon$ with $\epsilon \rightarrow 0$ to clarify that the beginning of this cut pinches the pole at the origin but the continuum contribution to the spectral density (discontinuity) vanishes at the position of the pole at $s=0$.

The residue $Z_{s}$ has a very clear interpretation, it is the "wave function renormalization" and its effect can be understood in two alternative manners. 
Consider the case in which $\tilde{J}=0$ in eqn.(48). Performing the inverse Laplace transform and invoking the Riemann-Lebesgue lemma, the long time behavior will be completely dominated by the pole at $s=0$. Therefore, if the velocity of the kink has been changed at $t=0$ by some external source, this disturbance will relax in time to an asymptotic value given by

$$
v_{\infty}=Z_{s} v_{0}
$$

Alternatively, consider the case of $v_{0}=0$ but with an external source term switched on at $t=0$ and constant in time thereafter. Again the inverse Laplace transform at long time will be dominated by the pole, and we find that the kink moves with constant acceleration given by

$$
\dot{v}=\frac{\tilde{j}}{M_{e f f}} \quad \text { with } \quad M_{e f f}=\frac{M}{Z_{s}} .
$$

Thus the wave function renormalization can also be understood as a renormalization of the kink mass. The ratio of the asymptotic acceleration to the initial acceleration is given by $Z_{s}$. As the kink moves, the interaction with the meson bath "dress" it changing its effective mass, which will be seen in specific models to be larger than the bare mass.

Furthermore we can now derive the following important sum rule. Consider the case $j \equiv 0$. Isolating the contribution from the pole and the cuts (by replacing $\delta\left(p_{o}-\omega_{p}+\omega_{k}\right) \rightarrow \delta\left(p_{o}-\omega_{p}+\omega_{k}-\epsilon\right)$ and taking the limit $\epsilon \rightarrow 0$ at the end of the computation) separating the pole and continuum contributions, and deforming the contour of integration for the inverse Laplace transform as shown in fig. (ब) we find the time evolution (for $j=0$ ) to be given by

$$
\frac{v(t)}{v_{0}}=Z_{s}+\frac{2}{\pi M} \int_{\epsilon}^{\infty} \frac{d \omega}{\omega} \frac{\left[-\tilde{\Gamma}_{I m}(\omega)\right] \cos (\omega t)}{\left[1+\frac{\tilde{\Gamma}_{R m}(\omega)}{M}\right]^{2}+\left[\frac{\tilde{\Gamma}_{I m}(\omega)}{M}\right]^{2}}
$$

Evaluating at $t=0$ we obtain the sum rule

$$
Z_{s}+\frac{2}{\pi M} \int_{\epsilon}^{\infty} \frac{d \omega}{\omega} \frac{\left[-\tilde{\Gamma}_{\operatorname{Im}}(\omega)\right]}{\left[1+\frac{\tilde{\Gamma}_{R m}(\omega)}{M}\right]^{2}+\left[\frac{\tilde{\Gamma}_{I m}(\omega)}{M}\right]^{2}}=1
$$

Since the spectral density $\rho(\omega)$ is positive (semi)definite as it will be explicitly shown below for specific models, the sum rule above determines that

$$
Z_{s}<1 \Longrightarrow \frac{v_{\infty}}{v_{0}}<1
$$

Although a sum rule similar to eqn. (60) is obtained in quantum field theory from the canonical commutation relations, its validity for the collective coordinate associated with the domain wall is far from obvious since the kinematic and canonical momentum conjugate to the collective coordinate are different. 
The continuum contribution in eqn.(59) is dominated at long times by the small $\omega$ region. Therefore for $T \neq 0$ the asymptotic long time relaxation of the velocity is completely determined by the Landau damping cut which has support at small $\omega$, whereas the two-meson cut vanishes below the threshold at $2 \omega_{0}$.

A further understanding of the dynamics will necessarily require knowledge of the matrix elements to establish the details of the kernels. This will be studied in particular models in the next section.

\subsection{Semiclassical Langevin equation}

The classical Langevin equation is an adequate phenomenological description of Brownian motion obtained by considering the dynamics of one (or few) degrees of freedom that interact with a bath in equilibrium.

It contains a term proportional to the velocity of the particle which incorporates friction and dissipation and a stochastic term which reflects the random interaction of the heat bath with the particle. These two terms are related by the classical fluctuation-dissipation relation which is derived in appendix 9 (see eqn. (130).

At the quantum mechanical level it is also possible to obtain a "reduced" or coarse grained description of the dynamics of one (or few) degrees of freedom in interaction with a bath. The coarse graining procedure has a very precise meaning: the full time dependent density matrix is traced over the bath degrees of freedom yielding an effective or "reduced" density matrix for the degrees of freedom whose dynamics is studied.

Such a description of non-equilibrium dynamics of a quantum mechanical particle coupled to a dissipative environment by a Langevin equation was presented by Caldeira and Leggett 30] and by Schmid [31]. Their technique is based on the influence-functional method of Feynman and Vernon [29] that naturally leads to a semiclassical Langevin equation.

In this section we follow the procedure of [30]-33] generalized to our case to derive the Langevin equation for kinks in a heat bath to lowest order in the adiabatic (weak) coupling.

The main step is to perform the path integrals over the meson degrees of freedom, thus obtaining an effective functional for the collective coordinate of the kink. Unlike the most usually studied cases of a particle linearly coupled to an harmonic reservoir 30]-34] we have here a bilinear coupling to the mesons. Therefore the influence functional cannot be obtained exactly, but it can be obtained in a consistent perturbative expansion. For this we treat the interaction term $\mathcal{L}_{I}\left[\dot{x}^{ \pm}, \gamma^{ \pm}, \gamma^{* \pm}\right]$ in perturbation theory up to second order in the vertex proportional to $\dot{x}^{ \pm}$(which is equivalent to lowest order in the adiabatic coupling $m / M$ ). Integrating over the meson variables and using 
$<F\left[a^{\dagger}, a\right]>=0$, we obtain

$$
\mathcal{Z}\left[j^{+}, j^{-}=0\right]=\int \mathcal{D} x^{+} \mathcal{D} x^{-} e^{i \int_{-\infty}^{\infty} d t^{\prime}\left(\mathcal{L}_{0}\left[\dot{x}^{+}\right]-\mathcal{L}_{0}\left[\dot{x}^{-}\right]\right)} \mathcal{F}\left[\dot{x}^{+}, \dot{x}^{-}\right],
$$

where

$$
\mathcal{L}_{0}\left[\dot{x}^{ \pm}\right]=\frac{1}{2} M\left(\dot{x}^{ \pm}\right)^{2}-j x^{ \pm}
$$

and $\mathcal{F}\left[\dot{x}^{+}, \dot{x}^{-}\right]$is the influence functional[29]-[34]. To lowest adiabatic order we find

$$
\begin{aligned}
\mathcal{F}\left[\dot{x}^{+}, \dot{x}^{-}\right]=\exp \left\{-\frac{1}{2} \int d t d t^{\prime}\right. & {\left[\dot{x}^{+}(t) G^{++}\left(t, t^{\prime}\right) \dot{x}^{+}\left(t^{\prime}\right)+\dot{x}^{-}(t) G^{--}\left(t, t^{\prime}\right) \dot{x}^{-}\left(t^{\prime}\right)\right.} \\
+ & \left.\left.\dot{x}^{+}(t) G^{+-}\left(t, t^{\prime}\right) \dot{x}^{-}\left(t^{\prime}\right)+\dot{x}^{-}(t) G^{-+}\left(t, t^{\prime}\right) \dot{x}^{+}\left(t^{\prime}\right)\right]\right\}
\end{aligned}
$$

in terms of the real-time meson correlation functions (see appendix $(\mathbb{B})$ )

$$
\begin{aligned}
& G^{++}\left(t, t^{\prime}\right)=<F\left[a^{\dagger+}(t), a^{+}(t)\right] F\left[a^{\dagger+}\left(t^{\prime}\right), a^{+}\left(t^{\prime}\right)\right]> \\
& G^{--}\left(t, t^{\prime}\right)=<F\left[a^{\dagger-}(t), a^{-}(t)\right] F\left[a^{\dagger-}\left(t^{\prime}\right), a^{-}\left(t^{\prime}\right)\right]> \\
& G^{+-}\left(t, t^{\prime}\right)=-<F\left[a^{\dagger+}(t), a^{+}(t)\right] F\left[a^{\dagger-}\left(t^{\prime}\right), a^{-}\left(t^{\prime}\right)\right]> \\
& G^{-+}\left(t, t^{\prime}\right)=-<F\left[a^{\dagger-}(t), a^{-}(t)\right] F\left[a^{\dagger+}\left(t^{\prime}\right), a^{+}\left(t^{\prime}\right)\right]>.
\end{aligned}
$$

At this stage it is convenient to introduce the center of mass and relative coordinates, $x$ and $R$ respectively, which are defined as

$$
x(t)=\frac{1}{2}\left(x^{+}(t)+x^{-}(t)\right) \quad, \quad R(t)=x^{+}(t)-x^{-}(t) .
$$

These are recognized as the coordinates used in the Wigner transform of the density matrix [30]- 34] in terms of which the partition function becomes

$$
\mathcal{Z}[0]=\int \mathcal{D} x \mathcal{D} R e^{i S[x, R]}
$$

with the non-equilibrium effective action given by

$$
S[x, R]=\int d t R(t)\left[-M \ddot{x}(t)-\frac{i}{2} \int d t^{\prime}\left(K_{1}\left(t-t^{\prime}\right) \dot{x}\left(t^{\prime}\right)-K\left(t-t^{\prime}\right) R\left(t^{\prime}\right)\right)\right]
$$

in terms of the kernels $K_{1}\left(t-t^{\prime}\right)$ and $K\left(t-t^{\prime}\right)$ which are given by (see appendix (B) )

$$
\begin{aligned}
K_{1}\left(t-t^{\prime}\right)= & 8 i \theta\left(t-t^{\prime}\right) \sum_{p, k \neq 0}\left\{T_{p k}^{(S)} T_{-p-k}^{(S)}\left(1+n_{p}+n_{k}\right)\left(\omega_{p}+\omega_{k}\right) \cos \left[\left(\omega_{p}+\omega_{k}\right)\left(t-t^{\prime}\right)\right]\right. \\
& \left.-T_{p k}^{(A)} T_{-p-k}^{(A)}\left(n_{p}-n_{k}\right)\left(\omega_{p}-\omega_{k}\right) \cos \left[\left(\omega_{p}-\omega_{k}\right)\left(t-t^{\prime}\right)\right]\right\} \\
= & -2 i \Sigma_{m}\left(t-t^{\prime}\right)
\end{aligned}
$$


and

$$
\begin{aligned}
K\left(t-t^{\prime}\right)= & -2 \sum_{p, k \neq 0}\left\{T_{p k}^{(S)} T_{-p-k}^{(S)}\left(1+n_{p}+n_{k}+n_{p} n_{k}\right)\left(\omega_{p}+\omega_{k}\right)^{2} \cos \left[\left(\omega_{p}+\omega_{k}\right)\left(t-t^{\prime}\right)\right]\right. \\
& \left.+2 T_{p k}^{(A)} T_{-p-k}^{(A)} n_{k}\left(1+n_{p}\right)\left(\omega_{p}-\omega_{k}\right)^{2} \cos \left[\left(\omega_{p}-\omega_{k}\right)\left(t-t^{\prime}\right)\right]\right\} .
\end{aligned}
$$

At this stage it proves convenient to introduce the identity

$$
e^{-\frac{1}{2} \int d t d t^{\prime} R(t) K\left(t-t^{\prime}\right) R\left(t^{\prime}\right)}=C(t) \int \mathcal{D} \xi e^{-\frac{1}{2} \int d t d t^{\prime} \xi(t) K^{-1}\left(t-t^{\prime}\right) \xi\left(t^{\prime}\right)+i \int d t \xi(t) R(t)}
$$

with $\mathrm{C}(\mathrm{t})$ being an inessential normalization factor, to cast the non-equilibrium effective action of the collective coordinate in terms of a stochastic noise variable with a definite probability distribution 31. 34].

$$
\mathcal{Z}[0]=\int \mathcal{D} x \mathcal{D} R \mathcal{D} \xi P[\xi] \exp \left\{i \int d t R(t)\left[-M \ddot{x}(t)-\frac{i}{2} \int d t^{\prime} K_{1}\left(t-t^{\prime}\right) \dot{x}\left(t^{\prime}\right)+\xi(t)\right]\right\}
$$

where the probability distribution of the stochastic noise, $P[\xi]$, is given by

$$
P[\xi]=\int \mathcal{D} \xi \exp \left\{-\frac{1}{2} \int d t d t^{\prime} \xi(t) K^{-1}\left(t-t^{\prime}\right) \xi\left(t^{\prime}\right)\right\} .
$$

In this approximation we find that the noise is Gaussian, additive and with correlation function given by

$$
<\xi(t) \xi\left(t^{\prime}\right)>=K\left(t-t^{\prime}\right)
$$

The semiclassical Langevin equation is obtained by extremizing the effective action in eqn.(72) with respect to $R(t)$ [30]-34]

$$
M \ddot{x}(t)+\int_{-\infty}^{t} d t^{\prime} \Sigma_{m}\left(t-t^{\prime}\right) \dot{x}\left(t^{\prime}\right)-j(t)=\xi(t) .
$$

Two features of the semiclassical Langevin equation deserve comment. The first is that the kernel $K_{1}\left(t-t^{\prime}\right)$, as can be seen from eqn.(69), is non-Markovian. The second is that the noise correlation function $K\left(t-t^{\prime}\right)$ is colored, i.e. it is not a delta function $\delta\left(t-t^{\prime}\right)$. The relationship between the kernels $K_{1}\left(t-t^{\prime}\right)$ and $K\left(t-t^{\prime}\right)$ established in appendix (ब) constitutes a generalized quantum fluctuation dissipation relation[30]-[34] (see eqn.(130). Finally we recognize that taking the average of (75) with the noise probability distribution $P[\xi]$ yields the equation of motion for the expectation value of the collective coordinate (eqn.(45)). 
A classical description is expected to emerge when the occupation distribution for the mesons can be approximated by their classical counterparts [30], i.e. when $n_{k} \approx T / \omega_{k}$.

If the kernels $\Sigma_{m}$ and $K$ admit a Markovian limit then a diffusion coefficient could be extracted by computing the long time limit of the correlation function $\left.<<(x(t)-x(0))^{2}\right\rangle>/ t$ where $\langle\langle\cdots\rangle\rangle$ stand for average over the noise distribution function. However when the kernels do not become Markovian, such a definition is not appropriate.

This summarizes the general formulation of the description of the dynamics of the collective coordinate both at the level of the evolution equation for the expectation value as well as for the effective Langevin dynamics in terms of stochastic noise terms arising from the fluctuations in the meson bath. We are now in condition to study specific models.

\section{TWO MODELS}

In the previous sections we established the general aspects of the real-time dynamics of kinks in the presence of the meson bath, obtaining the equation of motion as well as the Langevin equation for the collective coordinate in lowest adiabatic order. Further progress in the understanding of the dynamics necessarily involves the details of particular models which determine the matrix elements

$T^{(A, S)}$ and therefore the time dependence of the kernels involved. In this section we study these details for the Sine-Gordon and $\phi^{4}$ models.

\subsection{Sine-Gordon}

For the Sine-Gordon model the potential is given by

$$
U(\phi)=\frac{m^{2}}{\lambda}(1-\cos [\sqrt{\lambda} \phi])
$$

and the static kink solution is given by 17, 16, 26]

$$
\phi_{s}(x)=\frac{4}{\sqrt{\lambda}} \arctan \left[e^{m x}\right] .
$$

the kink mass and the adiabatic ratio are given by

$$
M=\frac{8 m}{\lambda} ; \frac{m}{M}=\frac{\lambda}{8}
$$

The normal modes of this theory are the solutions of the following equation, (see eqn.(66))

$$
\left[-\frac{d^{2}}{d x^{2}}+m^{2}-\frac{2 m^{2}}{\cosh ^{2}(m x)}\right] \psi_{n}(x)=\omega_{n}^{2} \psi_{n}(x) .
$$


The solutions of the above differential equation are well known [45], 17], 26]. There is only one bound state with zero eigenvalue, the zero mode, followed by a continuum with wave functions given by

$$
\mathcal{U}_{k}(x)=\frac{1}{\sqrt{2 \pi} \omega_{k}}(-i k+m \tanh (m x)) e^{i k x}
$$

with $\omega_{k}^{2}=k^{2}+m^{2}$, i.e. $w_{0}=m$. The scattering states represent the meson excitations around the $\operatorname{kink}[18]$.

The matrix elements $D_{p k}$ were already calculated by de Vega [26], (see eqns. 29. 16])) and are given by

$$
D_{k p}=i p \delta(k+p)+\frac{i\left(p^{2}-k^{2}\right)}{4 \omega_{k} \omega_{p} \sinh \left[\frac{\pi}{2} \frac{(p+k)}{m}\right]} \quad \text { for } p \neq k
$$

which determine the symmetric and antisymmetric matrix elements

$$
\begin{aligned}
& T_{p q}^{(S)}=\frac{1}{4}\left[\left(\frac{\omega_{p}}{\omega_{q}}\right)^{1 / 2}-\left(\frac{\omega_{q}}{\omega_{p}}\right)^{1 / 2}\right]\left\{\frac{\left(q^{2}-p^{2}\right)}{4 \omega_{k} \omega_{p} \sinh \left[\frac{\pi}{2} \frac{(q+p)}{m}\right]}\right\} \\
& T_{p q}^{(A)}=\frac{1}{4}\left[\left(\frac{\omega_{p}}{\omega_{q}}\right)^{1 / 2}+\left(\frac{\omega_{q}}{\omega_{p}}\right)^{1 / 2}\right]\left\{\frac{\left(q^{2}-p^{2}\right)}{4 \omega_{k} \omega_{p} \sinh \left[\frac{\pi}{2} \frac{(q+p)}{m}\right]} .\right.
\end{aligned}
$$

Since in this theory there are no bound states other than the zero mode, $F\left[a^{\dagger}, a\right]$ is given only by the first two terms in eqn.(123). We recognize the "structure factor"

$$
S(Q)=\int_{-\infty}^{\infty} \frac{d x}{2 \pi} e^{i Q x} \frac{2 m^{2}}{\cosh ^{2}[m x]}=\frac{Q}{\sinh \left[\frac{Q \pi}{2 m}\right]}
$$

This structure factor will play an important role in understanding the large energy behavior of the one-loop contribution. The important point to notice is that the structure factor is dominated by momenta $Q \approx m$, falling off exponentially for $|Q| \gg m$.

Substituting eqn.(82) in eqns.(41) and eqn.(44), we obtain the final form of the kernels in this case

$$
\begin{aligned}
& \Gamma_{m}\left(t-t^{\prime}\right)= \frac{1}{4^{3}} \int_{-\infty}^{\infty} d Q|S(Q)|^{2} \int_{-\infty}^{\infty} d k \frac{(Q-2 k)^{2}}{\omega_{Q-k}^{3} \omega_{k}^{3}}\left\{\left(1+2 n_{k}\right)\left(\omega_{Q-k}-\omega_{k}\right)^{2} \sin \left[\left(\omega_{Q-k}+\omega_{k}\right)\left(t-t^{\prime}\right)\right]\right. \\
&\left.-\quad\left(n_{Q-k}-n_{k}\right)\left(\omega_{Q-k}+\omega_{k}\right)^{2} \sin \left[\left(\omega_{Q-k}-\omega_{k}\right)\left(t-t^{\prime}\right)\right]\right\} \\
& \Sigma_{m}\left(t-t^{\prime}\right)= \frac{1}{4^{3}} \int_{-\infty}^{\infty} d Q|S(Q)|^{2} Q \int_{-\infty}^{\infty} d k \frac{(Q-2 k)^{3}}{\omega_{Q-k}^{3} \omega_{k}^{3}}\left\{\left(1+n_{Q-k}+n_{k}\right)\left(\omega_{Q-k}-\omega_{k}\right) \times\right. \\
&\left.\cos \left[\left(\omega_{Q-k}+\omega_{k}\right)\left(t-t^{\prime}\right)\right]-\left(n_{Q-k}-n_{k}\right)\left(\omega_{Q-k}+\omega_{k}\right) \cos \left[\left(\omega_{Q-k}-\omega_{k}\right)\left(t-t^{\prime}\right)\right]\right\} .
\end{aligned}
$$


The introduction of $S(Q)$ clarifies that $Q$ is the momentum transferred into the meson loop, and because $S(Q)$ is peaked at $Q=0$ with a width of the order of the meson mass we conclude that the momentum transferred into the meson loop is of the order of the meson mass. This observation will prove to be very important in the analysis of the high temperature limit in a later section.

It proves useful to express $\Gamma_{m}\left(t-t^{\prime}\right)$ and $\Sigma_{m}\left(t-t^{\prime}\right)$ in terms of dimensionless quantities to display at once the nature of the adiabatic expansion. To achieve this let us make the following change of variables

$$
Q \rightarrow \frac{Q}{m} \quad ; \quad k \rightarrow \frac{k}{m} \quad ; \quad \tau=m t \quad \text { and } \quad \mathcal{T}=\frac{T}{m} .
$$

Then $\Gamma_{m}\left(t-t^{\prime}\right)$ and $\Sigma_{m}\left(t-t^{\prime}\right)$ can be written as

$$
\Gamma_{m}\left(t-t^{\prime}\right)=m^{2} \Gamma\left(\tau-\tau^{\prime}\right) \quad \text { and } \quad \Sigma_{m}\left(t-t^{\prime}\right)=m^{3} \Sigma\left(\tau-\tau^{\prime}\right),
$$

where

$$
\begin{gathered}
\Gamma(\tau)=\int_{-\infty}^{\infty} d Q d k|S(Q)|^{2}\left\{\Gamma_{1}(Q, k) \sin \left[\left(w_{Q-k}+w_{k}\right)(\tau)\right]+\Gamma_{2}(Q, k) \sin \left[\left(w_{Q-k}-w_{k}\right)(\tau)\right]\right\} \\
\Sigma(\tau)=\int_{-\infty}^{\infty} d Q d k|S(Q)|^{2}\left\{\Sigma_{1}(Q, k) \cos \left[\left(w_{Q-k}+w_{k}\right)(\tau)\right]+\Sigma_{2}(Q, k) \cos \left[\left(w_{Q-k}-w_{k}\right)(\tau)\right]\right\}
\end{gathered}
$$

with

$$
\begin{aligned}
\Gamma_{1}(Q, k) & =\frac{1}{64} \frac{\left(1+2 n_{k}\right)(Q-2 k)^{2}\left(w_{Q-k}-w_{k}\right)^{2}}{w_{Q-k}^{3} w_{k}^{3}} \\
\Gamma_{2}(p, k) & =\frac{1}{32} \frac{n_{k}(Q-2 k)^{2}\left(w_{Q-k}+w_{k}\right)^{2}}{w_{Q-k}^{3} w_{k}^{3}} \\
\Sigma_{1}(Q, k) & =\left(w_{Q-k}+w_{k}\right) \Gamma_{1}(Q, k) \\
\Sigma_{2}(p, k) & =\left(w_{Q-k}-w_{k}\right) \Gamma_{2}(Q, k) \\
w_{k}^{2} & =k^{2}+1 ; n_{k}=\frac{1}{e^{\frac{w_{k}}{T}}-1} .
\end{aligned}
$$

Fig.(C) shows the numerical evaluation of $\Gamma(\tau)$ and $\Sigma(\tau)$ vs. $\tau$ for different values of $\mathcal{T}$. We clearly see that the self-energy kernel $\Sigma$ is peaked near $\tau=0$ and localized within a time scale $\tau_{s} \approx m^{-1}$. Similarly, the kernel $\Gamma$ varies slowly over a large time scale $\approx 5-10 m^{-1}$.

\subsubsection{Equation of motion: Exact solution vs. Markovian approximation}

In terms of dimensionless quantities the equation of motion (45) becomes in this case

$$
\dot{v}(\tau)+\frac{\lambda}{8} \int_{0}^{\tau} \mathrm{d} \tau^{\prime} \Sigma\left(\tau-\tau^{\prime}\right) v\left(\tau^{\prime}\right)=J
$$


where $J=j /(m M)$ and the dot stands for derivative with respect to the dimensionless variable $\tau$.

As shown in fig. ( $\mathrm{G})$, the kernel $\Sigma(\tau)$ has "memory" on time scales a few times the inverse of the meson frequency. If the velocity of the domain wall varies on time scales larger than the "memory" of the kernel a Markovian approximation to the dynamics may be reasonable. The first step in the Markovian approximation corresponds to replacing $v\left(\tau^{\prime}\right)$ by $v(\tau)$ inside the integral in eqn. (91) and taking it outside the integral. A second stage of approximation would take the upper limit of the integral to $\infty$ thus integrating the peak of the kernel. However we have shown above that the total integral of the kernel vanishes, thus this second stage cannot be invoked. Recognizing that $\int_{0}^{\tau} \Sigma\left(\tau-\tau^{\prime}\right) d \tau^{\prime}=\Gamma(\tau)$ the Markovian approximation to (91) is given by

$$
\dot{v}(\tau)+\frac{\lambda}{8} v(\tau) \Gamma(\tau)=J
$$

As advanced in the previous section, we now identify the kernel $\Gamma(\tau)$ as the dynamical friction coefficient in the Markovian approximation. The property (46) determines that $\Gamma(\tau \rightarrow \infty)=0$.

We will now focus on the initial value problem with $J=0$ and $v(\tau=0)=v_{0}$. The formal solution of the equation of motion in the Markovian approximation is given by

$$
v(\tau)=v_{0} e^{-\frac{\lambda}{8} \int_{0}^{\tau} \Gamma\left(\tau^{\prime}\right) d \tau^{\prime}}
$$

Even in the Markovian approximation the relaxation of the velocity at long times is not exponential because $\Gamma(\tau) \rightarrow 0$ at long times as can be seen in fig. (而).

\subsubsection{Velocity relaxation and wave function renormalization}

In order to display more clearly the dissipative effects, we now study the relaxation of the kink velocity. For this consider the initial value problem with $j(t>0)=0$ and initial velocity $v(t=$ $0)=v_{0}$.

As the kink moves through the bath, its velocity decreases because of the interaction with the mesons, the asymptotic final velocity is related to the initial velocity through the wave function renormalization as explained section 3.2 above. We present the numerical solution of the homogeneous equation for $v(t) / v_{0}$ in figure $(\overline{\mathrm{G}})$, where we also present the homogeneous solution in the Markovian approximation described above. We clearly see that the initial velocity relaxes to an asymptotic value $v_{\infty} / v_{0}$. However the time dependence cannot be fit with an exponential. We can see that even at high temperatures the Markovian approximation grossly fails to describe the dynamics.

According to the analysis of the general solution, the ratio $v_{\infty} / v_{0}$ should be given by the wave 
function renormalization, i.e.

$$
Z_{s}=\frac{1}{1+\frac{m}{M} \tilde{\Gamma}(s=0)}=\frac{v_{\infty}}{v_{0}}
$$

Table 1 below compares the ratio $v_{\infty} / v_{0}$ obtained from the numerical solution to the exact evolution equation, with the value of the wave-function renormalization. Clearly the agreement is excellent, confirming the analysis of the asymptotic behavior of the solution in real time.

Table (1): Numerical evaluation of $Z_{s}$ and $v_{\infty} / v_{0}$ in Sine-Gordon theory for $m / M=0.1,0.25$.

\begin{tabular}{||c||c|c||c|c||}
\hline \hline \multicolumn{1}{||c||}{} & \multicolumn{2}{c||}{$v_{\infty} / v_{0}$} & \multicolumn{2}{c||}{$Z_{s}$} \\
\hline & $\mathrm{m} / \mathrm{M}=0.1$ & $\mathrm{~m} / \mathrm{M}=0.25$ & $\mathrm{~m} / \mathrm{M}=0.1$ & $\mathrm{~m} / \mathrm{M}=0.25$ \\
\hline \hline Zero Temp. & 0.999808 & 0.999521 & 0.999808 & 0.999521 \\
\hline Temp. 1.0 & 0.993438 & 0.983754 & 0.993438 & 0.983753 \\
\hline Temp. 5.0 & 0.96055 & 0.906885 & 0.96055 & 0.90687 \\
\hline Temp. 10.0 & 0.923458 & 0.828352 & 0.923446 & 0.828303 \\
\hline \hline
\end{tabular}

\subsubsection{Kernels for the semiclassical Langevin equation}

Knowledge of the matrix elements $T^{(A)}, T^{(S)}$ allow us to obtain the final form of the kernels that enter in the semiclassical Langevin equation given by eqns.(69) and (70), and eqn.(82). These kernels can be written in terms of the dimensionless quantities given by eqn.(86). Since $K_{1}\left(t-t^{\prime}\right)=$ $-2 i \Sigma_{m}\left(t-t^{\prime}\right)$ we focus on $K\left(t-t^{\prime}\right)$. In term of dimensionless quantities, $K(t)=m^{4} \mathcal{K}(\tau)$ where

$$
\mathcal{K}(\tau)=\int_{-\infty}^{\infty} d Q d k Q^{2}|S(Q)|^{2}\left\{C_{1}(Q, k) \cos \left[\left(w_{Q-k}+w_{k}\right)(\tau)\right]+C_{2}(Q, k) \cos \left[\left(w_{Q-k}-w_{k}\right)(\tau)\right]\right\}
$$

with

$$
\begin{aligned}
C_{1}(Q, k) & =\frac{2}{4^{4}} \frac{\left(1+n_{Q-k}+n_{k}+n_{Q-k} n_{k}\right)(Q-2 k)^{4}}{w_{Q-k}^{3} w_{k}^{3}} \\
C_{2}(Q, k) & =\frac{1}{4^{3}} \frac{n_{k}\left(1+n_{Q-k}\right)(Q-2 k)^{4}}{w_{Q-k}^{3} w_{k}^{3}}
\end{aligned}
$$

The contribution from $C_{1}$ is recognized to arise from the process of emission and annihilation (spontaneous and induced) of two mesons, whereas that from $C_{2}$ arises from the scattering off in medium mesons and has its origin in the Landau damping diagram shown in figure (ब).

Fig.(C) shows $\mathcal{K}(\tau)$ for different temperatures $\mathcal{T}$. Notice that at large temperatures the kernel becomes strongly peaked at $\tau=0$ and one would be tempted to conclude that the classical limit 
corresponds to a delta function. However the coefficients (96,97) are such that the total integral in $\tau$ (leading to delta functions of sums and differences of frequencies) vanishes. We then conclude that even in the high temperature limit the noise-noise correlation function is not a delta function, i.e. the noise is "colored", the classical fluctuation dissipation relation in terms of a delta function noise correlation does not emerge and a diffusion coefficient cannot be appropriately defined. We postpone until a later section a discussion of the high temperature limit and the classical regime.

\section{$4.2 \quad \phi^{4}$ Theory}

In this model the potential is given by

$$
U(g, \phi)=\frac{m^{2}}{2 \lambda}\left(1-\lambda \phi^{2}\right)^{2}
$$

The static kink solution is given by

$$
\phi_{s}\left(x-x_{0}\right)=\frac{1}{\sqrt{\lambda}} \tanh \left[m\left(x-x_{0}\right)\right],
$$

and the kink mass is given by

$$
M=\frac{4 m}{3 \lambda}
$$

and the normal modes are the solutions to the equation, (see eqn.(6))

$$
\left[-\frac{d^{2}}{d x^{2}}+4 m^{2}-\frac{6 m^{2}}{\cosh ^{2}(m x)}\right] \psi_{n}(x)=\omega_{n}^{2} \psi_{n}(x) .
$$

The solution of the above differential equation is well known [45, [17]. It has two bound states followed by a continuum. The normalized eigenvectors are given by

$$
\begin{aligned}
\mathcal{U}_{0}(x) & =\frac{\sqrt{3 m}}{2} \operatorname{sech}^{2}[m x] \propto \frac{d \phi_{s}}{d x} \quad \text { with } \quad \omega_{0}=0 \\
\mathcal{U}_{b}(x) & =\frac{\sqrt{3 m}}{2} \operatorname{sech}[m x] \tanh [m x] \quad \text { with } \quad \omega_{b}^{2}=3 m^{2} \\
\mathcal{U}_{k}(x) & =\frac{m^{2} e^{i k x}}{\sqrt{2 \pi\left(k^{2}+m^{2}\right)} \omega_{k}}\left\{3 \tanh ^{2}[m x]-3 i \frac{k}{m} \tanh [m x]-1-\frac{k^{2}}{m^{2}}\right\}
\end{aligned}
$$

with $\omega_{k}^{2}=k^{2}+4 m^{2}$. The scattering states are identified with meson modes and the meson frequency is identified with $\omega_{o}=2 m$.

The bound state with zero frequency is the "zero mode", whereas the bound state with $\omega_{b}^{2}=3 m^{2}$ corresponds to an amplitude distortion [18, 16] or excited state of the kink.

The matrix elements $D_{p k}$ are given by, (see eqns.(29,16)) 


$$
\begin{array}{ll}
D_{b k}=\frac{\sqrt{3 \pi}}{8} \frac{\operatorname{sech}\left[\frac{\pi k}{2 m}\right]}{m^{\frac{3}{2}} \omega_{k}} \sqrt{k^{2}+m^{2}}\left(k^{2}+3 m^{2}\right) & \text { (from the bound state) } \\
D_{p k}=i k \delta(p+k)+\frac{3 i \pi\left(k^{2}-p^{2}\right)\left(p^{2}+k^{2}+4 m^{2}\right)}{4 m^{4} N_{p} N_{k} \sinh \left[\frac{\pi}{2} \frac{(p+k)}{m}\right]} \quad \text { for } p \neq k, &
\end{array}
$$

where $N_{k}$ is defined as

$$
N_{k}=\sqrt{\frac{2 \pi w_{k}^{2}\left(k^{2}+m^{2}\right)}{m^{4}}} .
$$

We notice that the coupling to the continuum through the bound state given by the matrix element $D_{b k}$ is of the same order as the coupling to the continuum-continuum (matrix elements $\left.D_{p k}\right)$. This will have interesting consequences for the dissipational dynamics. The symmetric and antisymmetric matrix elements for the continuum states are given by

$$
\begin{aligned}
& T_{p q}^{(S)}=\frac{3}{32}\left[\left(\frac{\omega_{p}}{\omega_{q}}\right)^{1 / 2}-\left(\frac{\omega_{q}}{\omega_{p}}\right)^{1 / 2}\right]\left\{\frac{\left(q^{2}-p^{2}\right)\left(p^{2}+q^{2}+4 m^{2}\right)}{\sqrt{q^{2}+m^{2}} \sqrt{p^{2}+m^{2}} \omega_{q} \omega_{p} \sinh \left[\frac{\pi}{2} \frac{(q+p)}{m}\right]}\right\} \\
& T_{p q}^{(A)}=\frac{3}{32}\left[\left(\frac{\omega_{p}}{\omega_{q}}\right)^{1 / 2}+\left(\frac{\omega_{q}}{\omega_{p}}\right)^{1 / 2}\right]\left\{\frac{\left(q^{2}-p^{2}\right)\left(p^{2}+q^{2}+4 m^{2}\right)}{\sqrt{q^{2}+m^{2}} \sqrt{p^{2}+m^{2}} \omega_{q} \omega_{p} \sinh \left[\frac{\pi}{2} \frac{(q+p)}{m}\right]},\right.
\end{aligned}
$$

whereas those involving the bound state are obtained by replacing the matrix elements $D_{b k}$ for the $D_{p k}$.

Since in this model there is one bound state other than the zero mode, the interaction vertex $F\left[a^{\dagger}, a\right]$ is given by eqn.(123) in the appendix. The contributions from bound-state-continuum virtual transitions do not mix with the continuum-continuum to this order in the adiabatic expansion. As a consequence of this simplification the dimensionless kernels (in terms of the dimensionless variables introduced in (86)) become

$$
\begin{aligned}
\Gamma(\tau)= & \int_{-\infty}^{\infty} \mathrm{d} p\left\{\Gamma_{1}^{b}(p) \sin \left[\left(w_{p}+w_{b}\right)(\tau)\right]+\Gamma_{2}^{b}(p) \sin \left[\left(w_{p}-w_{b}\right)(\tau)\right]\right. \\
& \left.+\int_{-\infty}^{\infty} d Q|S(Q)|^{2} \mathrm{~d} k\left\{\Gamma_{1}(Q, k) \sin \left[\left(w_{Q-k}+w_{k}\right)(\tau)\right]+\Gamma_{2}(Q, k) \sin \left[\left(w_{Q-k}-w_{k}\right)(\tau)\right]\right\}\right\} \\
\Sigma(\tau)= & \int_{-\infty}^{\infty} \mathrm{d} p\left\{\Sigma_{1}^{b}(p) \cos \left[\left(w_{p}+w_{b}\right)(\tau)\right]+\Sigma_{2}^{b}(p) \cos \left[\left(w_{p}-w_{b}\right)(\tau)\right]\right. \\
& \left.+\int_{-\infty}^{\infty} \mathrm{d} Q|S(Q)|^{2} \mathrm{~d} k\left\{\Sigma_{1}(Q, k) \cos \left[\left(w_{Q-k}+w_{k}\right)(\tau)\right]+\Sigma_{2}(Q, k) \cos \left[\left(w_{Q-k}-w_{k}\right)(\tau)\right]\right\}\right\}
\end{aligned}
$$


with

$$
\begin{aligned}
\Gamma_{1}(Q, k) & \equiv \frac{3^{2}}{4^{4}} \frac{\left(1+n_{Q-k}+n_{k}\right)(Q-2 k)^{2}\left(w_{Q-k}-w_{k}\right)^{2}\left((Q-k)^{2}+k^{2}+4\right)^{2}}{w_{Q-k}^{3} w_{k}^{3}\left((Q-k)^{2}+1\right)\left(k^{2}+1\right)} \\
\Gamma_{2}(Q, k) & \equiv \frac{3^{2}}{4^{4}} \frac{\left(n_{k}-n_{Q-k}\right)(Q-2 k)^{2}\left(w_{Q-k}+w_{k}\right)^{2}\left((Q-k)^{2}+k^{2}+4\right)^{2}}{w_{Q-k}^{3} w_{k}^{3}\left((Q-k)^{2}+1\right)\left(k^{2}+1\right)} \\
\Gamma_{1}^{b}(p) & \equiv \frac{\pi \sqrt{3}}{128} \frac{\left(p^{4}+4 p^{2}+3\right)^{2}\left(w_{p}-w_{b}\right)\left(1+n_{b}+n_{p}\right)}{w_{p}^{3}\left(w_{p}+w_{b}\right)} \operatorname{sech}^{2}\left[\frac{\pi p}{2}\right] \\
\Gamma_{2}^{b}(p) & \equiv \frac{\pi \sqrt{3}}{128} \frac{\left(p^{4}+4 p^{2}+3\right)^{2}\left(w_{p}+w_{b}\right)\left(n_{b}-n_{p}\right)}{w_{p}^{3}\left(w_{p}-w_{b}\right)} \operatorname{sech}^{2}\left[\frac{\pi p}{2}\right] \\
\Sigma_{1}(Q, k) & \equiv\left(w_{Q-k}+w_{k}\right) \Gamma_{1}(Q, k) ; \Sigma_{2}(Q, k)=\left(w_{Q-k}-w_{k}\right) \Gamma_{2}(Q, k) \\
\Sigma_{1}^{b}(p) & \equiv\left(w_{p}+w_{b}\right) \Gamma_{1}^{b}(p) ; \Sigma_{2}^{b}(p)=\left(w_{p}-w_{b}\right) \Gamma_{2}^{b}(p) \\
w_{k}^{2} & =k^{2}+4,
\end{aligned}
$$

where $\Sigma(\tau)$ and $\Gamma(\tau)$ are defined as in eqn.(87). The functions $\Sigma(\tau)$ and $\Gamma(\tau)$ where evaluated numerically at different temperatures $\mathcal{T}$, the results are displayed in fig.(可). The behavior of these functions differ from those in the Sine-Gordon theory because of the presence of the bound state which is interpreted as an excited state of the kink. As the kink moves in the dissipative medium, energy is transferred between the kink and the bound state resulting in the Rabi-like oscillations displayed in the figure. We notice that the contribution of the bound state is of the same order of magnitude as that of the continuum.

\subsubsection{Equation of motion: Exact solution vs. Markovian approximation}

The solution to the equation of motion and the comparison to the Markovian approximation proceeds just as in the the case of the Sine-Gordon model. The equation of motion is again solved as an initial value problem. The exact and Markovian solutions are displayed in figure (C).

The new feature of the solution are the oscillations that result from virtual transitions to the bound state. We interpret these in the following manner: as the kink moves it excites the bound state that corresponds to a kink distortion[18], this excitation in turn reacts-back in the dynamics of the collective coordinate in a retarded manner.

While the exact solution in this model is qualitatively similar to that of the Sine-Gordon model, we see however, that quantitatively they are different: there is stronger dynamical dissipation in the $\phi^{4}$ model as compared to the Sine Gordon case, due to the strong coupling to the bound state-continuum intermediate states. 


\subsubsection{Velocity relaxation and wave function renormalization}

In this model the Laplace transform of the functions $\Gamma(\tau)$ and $\Sigma(\tau)$ are given by

$$
\begin{aligned}
\tilde{\Sigma}(s)= & \int_{-\infty}^{\infty} \mathrm{d} Q|S(Q)|^{2} \mathrm{~d} k\left\{\frac{\Sigma_{1}(Q, k) s}{s^{2}+\left(w_{Q-k}+w_{k}\right)^{2}}+\frac{\Sigma_{2}(Q, k) s}{s^{2}+\left(w_{Q-k}-w_{k}\right)^{2}}\right\} \\
& +\int_{-\infty}^{\infty} \mathrm{d} p\left\{\frac{\Sigma_{1}^{b}(p) s}{s^{2}+\left(w_{p}+w_{b}\right)^{2}}+\frac{\Sigma_{2}^{b}(p) s}{s^{2}+\left(w_{p}-w_{b}\right)^{2}}\right\} \\
\tilde{\Sigma}(s) \equiv & s \tilde{\Gamma}(s) \\
\tilde{\Gamma}(s)= & \int_{-\infty}^{\infty} \mathrm{d} Q|S(Q)|^{2} \mathrm{~d} k\left\{\frac{\Gamma_{1}(Q, k)\left(w_{p}+w_{k}\right)}{s^{2}+\left(w_{Q-k}+w_{k}\right)^{2}}+\frac{\Gamma_{2}(Q, k)\left(w_{p}-w_{k}\right)}{s^{2}+\left(w_{Q-k}-w_{k}\right)^{2}}\right\} \\
& +\int_{-\infty}^{\infty} \mathrm{d} p\left\{\frac{\Gamma_{1}^{b}(p)\left(w_{p}+w_{b}\right)}{s^{2}+\left(w_{p}+w_{b}\right)^{2}}+\frac{\Gamma_{2}^{b}(p)\left(w_{p}-w_{b}\right)}{s^{2}+\left(w_{p}-w_{b}\right)^{2}}\right\} .
\end{aligned}
$$

With the quantities $\Sigma^{b} ; \Gamma^{b}$ given above. The homogeneous equations of motion given by (91) (exact) and its Markovian approximation (92) both with $j=0$ are solved with the kernels $\Sigma(\tau) ; \Gamma(\tau)$ given above for $v(t) / v_{0}$. The asymptotic behavior of the exact solution will be compared with the prediction $v_{\infty} / v_{0}=Z_{s}$, with the wave function renormalization $Z_{s}$ given by eqn.(94) but with the $\tilde{\Gamma}(s=0)$ appropriate to the $\phi^{4}$ model.

Figure (C) shows the numerical solutions of eqn.(91) and eqn.(92) with $j=0$ for $\left(v(t) / v_{0}\right)$ for temperatures $\mathcal{T}=0,1.0,5.0$ and 10.0. Again the Rabi-like oscillations associated with the excitation of the bound state is apparent in the solutions. We have checked numerically that asymptotically the velocity tends to a constant value $v_{\infty}$ but not exponentially. Table 2 shows the values of $v_{\infty} / v_{0}$ and $Z_{s}$ for these temperatures for $m / M=0.1$ and 0.25 where $v_{\infty} / v_{0}$ was evaluated at $\tau=200$ for the exact solution. Within our numerical errors, we can see that eqn.(94) is fulfilled.

Table (2): Numerical evaluation of $Z_{s}$ and $v_{\infty} / v_{0}$ in $\phi^{4}$ theory for $m / M=0.1,0.25$.

\begin{tabular}{||c||c|c||c|c||}
\hline \hline \multicolumn{1}{||c||}{} & \multicolumn{2}{c||}{$v_{\infty} / v_{0}$} & \multicolumn{2}{c||}{$Z_{s}$} \\
\hline & $\mathrm{m} / \mathrm{M}=0.1$ & $\mathrm{~m} / \mathrm{M}=0.25$ & $\mathrm{~m} / \mathrm{M}=0.1$ & $\mathrm{~m} / \mathrm{M}=0.25$ \\
\hline \hline Zero Temp. & 0.999225 & 0.998064 & 0.999176 & 0.997943 \\
\hline Temp. 1.0 & 0.961561 & 0.911004 & 0.96376 & 0.914072 \\
\hline Temp. 5.0 & 0.784934 & 0.593058 & 0.787734 & 0.597494 \\
\hline Temp. 10.0 & 0.642698 & 0.417231 & 0.646577 & 0.422562 \\
\hline \hline
\end{tabular}




\subsubsection{Kernels for the semiclassical Langevin equation}

From the definition of the kernels $K_{1}\left(t-t^{\prime}\right)$ and $K\left(t-t^{\prime}\right)$, eqns.(69) and (70), and eqn.(105), these kernels can be written in terms of the dimensionless quantities given by eqn. (86) as

$$
\mathcal{K}_{1}\left(\tau-\tau^{\prime}\right)=-2 i \Sigma\left(\tau-\tau^{\prime}\right),
$$

where $\Sigma\left(\tau-\tau^{\prime}\right)$ is given by eqn.106) and $K(t)=m^{4} \mathcal{K}(\tau)$ with

$$
\begin{aligned}
\mathcal{K}(\tau)= & \int_{-\infty}^{\infty} \mathrm{d} p\left\{C_{1}^{b}(p) \cos \left[\left(w_{p}+w_{b}\right)(\tau)\right]+C_{2}^{b}(p) \cos \left[\left(w_{p}-w_{b}\right)(\tau)\right]\right\}+ \\
& \int_{-\infty}^{\infty} \mathrm{d} Q|S(Q)|^{2} \int_{-\infty}^{\infty} \mathrm{d} k\left\{C_{1}(Q, k) \cos \left[\left(w_{Q-k}+w_{k}\right)(\tau)\right]+C_{2}(Q, k) \cos \left[\left(w_{Q-k}-w_{k}\right)(\tau)\right]\right\}
\end{aligned}
$$

with the dimensionless matrix elements

$$
\begin{aligned}
C_{1}(Q, k) & \equiv \frac{18}{4^{5}} \frac{Q^{2}\left(1+n_{Q-k}+n_{k}+n_{Q-k} n_{k}\right)(Q-2 k)^{4}\left((Q-k)^{2}+k^{2}+4\right)^{2}}{w_{Q-k}^{3} w_{k}^{3}\left((Q-k)^{2}+1\right)\left(k^{2}+1\right)} \\
C_{2}(Q, k) & \equiv \frac{9}{4^{4}} \frac{n_{k} Q^{2}\left(1+n_{Q-k}\right)(Q-2 k)^{4}\left((Q-k)^{2}+k^{2}+4\right)^{2}}{w_{Q-k}^{3} w_{k}^{3}\left((Q-k)^{2}+1\right)\left(k^{2}+1\right)} \\
C_{1}^{b}(p) & \equiv \frac{\pi \sqrt{3}}{4^{4}} \frac{\left(p^{4}+4 p^{2}+3\right)^{2}\left(p^{2}+1\right)\left(1+n_{b}+n_{p}+n_{b} n_{p}\right)}{w_{p}^{3} \cosh ^{2}\left[\frac{\pi p}{2}\right]} \\
C_{2}^{b}(p) & \equiv \frac{2 \pi \sqrt{3}}{4^{4}} \frac{\left(p^{4}+4 p^{2}+3\right)^{2}\left(p^{2}+1\right) n_{p}\left(1+n_{b}\right)}{w_{p}^{3} \cosh ^{2}\left[\frac{\pi p}{2}\right]} .
\end{aligned}
$$

Fig.(C) shows $\mathcal{K}(\tau)$ vs. $\tau$ for temperatures $\mathcal{T}=0,1,5,10$. Again the oscillations are a consequence of the bound state contribution, and as in the Sine-Gordon case we find that despite the fact that in the high temperature limit the kernel becomes very localized in time, the total integral $\int_{-\infty}^{\infty} d \tau \mathcal{K}(\tau)=0$ preventing a representation of the noise-noise correlation function as a delta function in time even in the high temperature limit. The "color" in the noise-noise correlation function is enhanced by the coupling to the continuum via the bound state which is also responsible for the strong oscillatory behavior of the real-time correlation function.

\section{HARD THERMAL LOOPS VS. CLASSICAL LIMIT}

The high temperature limit corresponds to $T>m$ with $m$ the meson mass. However we are restricted to the dilute limit in which the treatment of isolated domain walls is meaningful. Because the kink density is suppressed by an Arrhenius activation factor [8]

$$
N_{k} \approx e^{-\frac{M}{T}}
$$


the study of the high temperature limit for the dynamics of isolated domain walls requires that the temperature range be such that

$$
m<<T<<M \approx \frac{m}{\lambda}
$$

For weak coupling $\lambda<<1$ there is a wide temperature range in which the high temperature and the dilute kink gas approximation will be reliable. In order to understand the high temperature limit it is convenient to separate the loop integrals into the "soft part" in which both the integrated and transferred momenta are "soft", i.e. $k, Q<<T$ and the "hard" part, in which the loop momentum $k$ is $\mathcal{O}(T)$. Since the structure factor $S(Q)$ is strongly suppressed for $Q>>m$, the transferred momentum $Q$ is always of order of $m<<T$ hence it is always "soft". The "hard" $k$ region with "soft" transferred momentum is the domain of validity of the hard-thermal loop resummation programme of Braaten and Pisarski[48].

Then for the soft region of the remaining $k$ integral, we can replace the occupation factors $n_{k} \approx T / \omega_{k}$. This soft region therefore gives the classical contribution to the kernels $\Sigma, \Gamma$. A simple WKB analysis of the continuum solutions for both cases considered, reveals that the matrix elements $D_{k, p}$ fall off as $\approx S(p+k) / k$ in the limit in which $Q=k+p \approx m ; k \rightarrow \infty$. This simple analysis is confirmed by the exact expression for the matrix elements $T_{k, p}$ (see equations 82,105) which in this limit $(Q=k+p \approx m, k \rightarrow \infty)$ behave as $S(Q) / k$. Therefore in the meson loop, the matrix elements yield a contribution of $\mathcal{O}\left(1 / k^{2}\right)$ in the hard thermal loop limit, for which a simple scaling analysis reveals a large temperature behavior of $\mathcal{O}(1 / T)$. Hence we see that in the $1+1$ dimensional case the hard-thermal loop limit yields a subleading contribution as compared to the classical contribution from the soft region. This is a consequence of the small phase space available for the loop integrals in $1+1$ space-time dimensions.

This analysis allows us to conclude that the high temperature limit is dominated by the classical contribution with a lineal dependence on temperature in the regime $T>>m$. This behavior is clearly displayed in figure (C) that shows the integrals $I_{1} ; I_{2}$ with

$$
\begin{aligned}
& I_{1}=\int_{-\infty}^{\infty} d k \frac{(Q-2 k)^{3}\left(\omega_{Q-k}-\omega_{k}\right)}{\omega_{Q-k}^{3} \omega_{k}^{3}} n_{k} \\
& I_{2}=\int_{-\infty}^{\infty} d k \frac{(Q-2 k)^{3}\left(\omega_{Q-k}+\omega_{k}\right)}{\omega_{Q-k}^{3} \omega_{k}^{3}} n_{k},
\end{aligned}
$$

corresponding to the contributions from $\Sigma_{1} ; \Sigma_{2}$ to the self-energy kernel at $\tau=0$ for Sine Gordon theory, with similar results for $\phi^{4}$. We clearly see that for $T \geq 2 m$ the temperature dependence becomes lineal. Furthermore we have numerically checked that most of the contribution in this regime arises from the "soft" region of the loop momentum $k \leq m$ and $Q \leq m$.

Combined together the hard-thermal loop analysis and the numerical evidence lead us to conclude unambiguously that the high temperature limit of the self-energy kernel is dominated by the classical finite temperature contribution.

This analysis also holds for the noise-noise correlation function (since the same matrix elements 
contribute to them). However for the noise-noise correlation function there is an extra factor of the Bose occupation factors in the integrals. This results in one extra power of temperature in the "soft" region while the temperature dependence from the hard-thermal loop region is mostly unaffected by the extra Bose factor. Therefore we conclude that the self-energy kernel is $\mathcal{O}\left(m^{2} T\right)$ and the noise-noise correlation function is $\mathcal{O}\left(m^{2} T^{2}\right)$ in the high temperature limit. This is in accord with the classical Fluctuation-Dissipation theorem in which the noise-noise correlation function has an extra power of temperature compared with the dissipative contribution.

At long times the contribution from the two meson cut gives a rapidly oscillating contribution leading to a rapid fall off of the time dependence. On the other hand, the contribution from the Landau damping cut gives the leading contribution at long times because the discontinuity has support at very low frequencies and completely dominates long time behavior. Therefore we conclude that the long time, high temperature behavior in the dilute kink limit is completely dominated by classical finite temperature dynamics and dominated by the contribution from Landau damping.

\section{HIGHER ORDERS AND HIGHER DIMENSIONS}

At two-loops and higher orders we expect that collisions will provide a non vanishing static friction coefficient and result in an exponential relaxation of the velocity in some time regime. However, the contribution from these terms will be of higher order in coupling $(m / M)$ and therefore there will be a competition between the time scales associated with lowest order relaxation via off-shell Landau damping and the higher order collisional relaxation leading to an exponential fall-off. Therefore we anticipate several different relaxational regimes with wide separation of the time scales for weak couplings and in the dilute regime.

In $3+1$ dimensions for degenerate scalar potentials the situation is clearly more complicated. The zero mode from translation invariance now gives rise to two-dimensional massless degree of freedom corresponding to small local distortions perpendicular to the (planar) wall. These are the capillary waves fluctuations of the interface that will dominate the long-wavelength small frequency dynamics. We expect to report on further studies of higher order collisional relaxation as well as new phenomena in $3+1$ dimensions in the near future.

\section{CONCLUSIONS AND FURTHER QUESTIONS}

We have studied the non-equilibrium dynamics of domain walls in $1+1$ dimensional scalar field theories at finite temperature in the dilute regime. We obtained the real time equations of motion for the expectation value of the collective coordinate and also the quantum Langevin equation to lowest order in the weak coupling (adiabatic) expansion. Two specific models were studied: $\phi^{4}$ and 
Sine-Gordon scalar field theories providing detailed analytic and numerical studies of the equations of motion and a Markovian approximation to it.

To lowest order in weak coupling we found that the real-time equation of motion involves a non-Markovian self-energy kernel and that the static friction coefficient vanishes. However, there is dynamical friction which is a result of the memory effects in the self-energy and is associated with two different types of two-meson processes: spontaneous and induced two-meson creation and annihilation and scattering off in medium mesons. The second type processes only occur at finite temperature and lead to Landau damping.

We studied the Markovian approximation and shown numerically that this approximation is unreliable in a wide range of temperatures.

The quantum Langevin equation was obtained by computing the influence functional obtained by tracing out the meson degrees of freedom to the same order in the adiabatic expansion. We found that the dissipative kernel and the noise correlation function obey a generalized form of fluctuation-dissipation relation but that a Markovian limit is not available, the noise is Gaussian, additive but colored.

The high temperature limit in the dilute regime was studied in detail by analyzing the "soft" and "hard" contributions to the self energy and noise kernels. We find that the hard contribution is suppressed at high temperature because the matrix elements fall of as an inverse power of the hard momentum. The small one dimensional phase space leads to a suppression of the hard momenta and therefore the leading contribution at high temperature arises from the "soft" region with momenta of the order of the meson mass yielding the classical result in the high temperature limit. Furthermore the long time dynamics is completely determined by the Landau damping processes in the medium leading to the conclusion that to the order studied the long time dynamics is completely determined by classical Landau damping.

We have restricted our study to a perturbative expansion which already showed the complicated nature of the problem even at lowest order. Pursuing a higher order calculation in perturbation theory will clearly be a major task. When the velocity of the soliton becomes very large one must abandon the approach advocated in this article and pursue a non-perturbative approach that accounts for strongly non-linear processes. We are currently implementing such an approach in terms of a self-consistent variational method 49] and expect to report on new results in the near future.

\section{Acknowledgments}

The authors would like to thank D. Jasnow, H. de Vega, J. Levy, R. Willey and D. Campbell for helpful discussions and comments. D. B. thanks S. Khlebnikov and P. Arnold for bringing Refs. [14, 15] to his attention and for discussions. The authors also thank N.S.F. for partial support 
through grant awards: PHY-9605186, INT-9512798 and the Pittsburgh Supercomputing Center for grant: PHY-950011P. S. M. A. thanks King Fahad University of Petroleum and Minerals (Saudi Arabia) for financial support. F. I. T. thanks the Dept. of Physics, Univ. of Pittsburgh for hospitality and CNPq and FAPEMIG for financial support.

\section{A REAL-TIME MESON CORRELATION FUNCTIONS}

In this appendix, we will calculate the Green's functions which are defined in eqn.(65) in terms of the vertex given by eqn. (32).

Applying Wick's theorem and eqn.(38), it is a matter of straightforward algebra to find the following results:

$$
\begin{aligned}
G^{++}\left(t, t^{\prime}\right)= & -2 \sum_{p, k \neq 0}\left\{T _ { p k } ^ { ( S ) } T _ { - p - k } ^ { ( S ) } \left[e^{-i\left(\omega_{p}+\omega_{k}\right)\left(t-t^{\prime}\right)}\left(n_{p} n_{k}+\theta\left(t-t^{\prime}\right)\left(1+n_{p}+n_{k}\right)\right)\right.\right. \\
& \left.+\quad e^{+i\left(\omega_{p}+\omega_{k}\right)\left(t-t^{\prime}\right)}\left(n_{p} n_{k}+\theta\left(t^{\prime}-t\right)\left(1+n_{p}+n_{k}\right)\right)\right] \\
& \left.+2 T_{p k}^{(A)} T_{-p-k}^{(A)}\left[e^{-i\left(\omega_{p}-\omega_{k}\right)\left(t-t^{\prime}\right)}\left(n_{p} n_{k}+n_{p} \theta\left(t^{\prime}-t\right)+n_{k} \theta\left(t-t^{\prime}\right)\right)\right]\right\} \\
= & G^{>}\left(t, t^{\prime}\right) \theta\left(t-t^{\prime}\right)+G^{<}\left(t, t^{\prime}\right) \theta\left(t^{\prime}-t\right) \\
G^{--}\left(t, t^{\prime}\right)= & -2 \sum_{p, k \neq 0}\left\{T _ { p k } ^ { ( S ) } T _ { - p - k } ^ { ( S ) } \left[e^{-i\left(\omega_{p}+\omega_{k}\right)\left(t-t^{\prime}\right)}\left(n_{p} n_{k}+\theta\left(t^{\prime}-t\right)\left(1+n_{p}+n_{k}\right)\right)\right.\right. \\
& \left.\quad+2 T_{p k}^{(A)} T_{-p-k}^{(A)}\left[e^{-i\left(\omega_{p}-\omega_{k}\right)\left(t-t^{\prime}\right)}\left(n_{p} n_{k}+n_{k} \theta\left(t^{\prime}-t\right)+n_{p} \theta\left(t-t^{\prime}\right)\right)\right]\right\} \\
= & G^{>}\left(t, t^{\prime}\right) \theta\left(t^{\prime}-t\right)+G^{<}\left(t, t^{\prime}\right) \theta\left(t-t^{\prime}\right) \\
G^{+-}\left(t, t^{\prime}\right)= & \quad \sum_{p, k \neq 0}\left\{T_{p k}^{(S)} T_{-p-k}^{(S)}\left[e^{-i\left(\omega_{p}+\omega_{k}\right)\left(t-t^{\prime}\right)} n_{p} n_{k}+e^{+i\left(\omega_{p}+\omega_{k}\right)\left(t-t^{\prime}\right)}\left(n_{p} n_{k}+n_{p}+n_{k}+1\right)\right)\right] \\
& \left.+2 T_{p k}^{(A)} T_{-p-k}^{(A)}\left[e^{-i\left(\omega_{p}-\omega_{k}\right)\left(t-t^{\prime}\right)} n_{p}\left(1+n_{k}\right)\right]\right\}
\end{aligned}
$$




$$
\begin{aligned}
G^{-+}\left(t, t^{\prime}\right)= & 2 \sum_{p, k \neq 0}\left\{T_{p k}^{(S)} T_{-p-k}^{(S)}\left[e^{-i\left(\omega_{p}+\omega_{k}\right)\left(t-t^{\prime}\right)}\left(n_{p} n_{k}+n_{p}+n_{k}+1\right)+e^{+i\left(\omega_{p}+\omega_{k}\right)\left(t-t^{\prime}\right)} n_{p} n_{k}\right]\right. \\
& \left.+2 T_{p k}^{(A)} T_{-p-k}^{(A)}\left[e^{-i\left(\omega_{p}-\omega_{k}\right)\left(t-t^{\prime}\right)} n_{k}\left(1+n_{p}\right)\right]\right\} \\
= & -G^{>}\left(t, t^{\prime}\right)=-G^{<}\left(t^{\prime}, t\right) .
\end{aligned}
$$

These Green's functions satisfy the following relation

$$
G^{++}+G^{--}+G^{+-}+G^{-+}=0
$$

which is a consequence of unitary time evolution 42].

Furthermore, using the antisymmetry property of the matrix elements $T_{p k}^{(A)}$ one finds that

$$
G^{+-}\left(t, t^{\prime}\right)=\left(G^{-+}\left(t, t^{\prime}\right)\right)^{*}
$$

The Green's functions $G^{++}\left(t, t^{\prime}\right) ; G^{--}\left(t, t^{\prime}\right)$ can be written in terms of $G^{+-}\left(t, t^{\prime}\right)$ and its complex conjugate, therefore we see that there is only one independent Green's functions (and its complex conjugate).

\section{B CALCULATING $K_{1}\left(t-t^{\prime}\right)$ AND $K\left(t-t^{\prime}\right)$}

Performing the coordinate transformation in eqn.([66), the influence-functional becomes

$$
\begin{aligned}
\mathcal{F}[\dot{x}, \dot{R}] & =\exp \left\{-\frac{1}{2} \int d t d t^{\prime}\left[\frac{\dot{R}(t) \dot{R}\left(t^{\prime}\right)}{4}\left(G^{++}\left(t, t^{\prime}\right)+G^{--}\left(t, t^{\prime}\right)-G^{+-}\left(t, t^{\prime}\right)-G^{-+}\left(t, t^{\prime}\right)\right)\right.\right. \\
& +\left\{\frac{1}{2} \dot{R}(t) \dot{x}\left(t^{\prime}\right)\left(G^{++}\left(t, t^{\prime}\right)-G^{--}\left(t, t^{\prime}\right)+G^{+-}\left(t, t^{\prime}\right)-G^{-+}\left(t, t^{\prime}\right)\right)\right. \\
& \left.\left.\left.+\frac{1}{2} \dot{x}(t) \dot{R}\left(t^{\prime}\right)\left(G^{++}\left(t, t^{\prime}\right)-G^{--}\left(t, t^{\prime}\right)-G^{+-}\left(t, t^{\prime}\right)+G^{-+}\left(t, t^{\prime}\right)\right)\right\}\right]\right\} .
\end{aligned}
$$

Integrating the linear term in $\dot{R}$ by parts once and the quadratic term twice, the influencefunctional can be cast in the following form

$$
\mathcal{F}[\dot{x}, \dot{R}]=\exp \left\{\frac{1}{2} \int d t d t^{\prime}\left[R(t) K_{1}\left(t-t^{\prime}\right) \dot{x}\left(t^{\prime}\right)-R(t) K\left(t-t^{\prime}\right) \dot{R}\left(t^{\prime}\right)\right]\right\}
$$

where

$$
K_{1}\left(t-t^{\prime}\right)=\frac{1}{2} \frac{\partial}{\partial t}\left[\left(G^{++}\left(t, t^{\prime}\right)-G^{--}\left(t, t^{\prime}\right)+G^{+-}\left(t, t^{\prime}\right)-G^{-+}\left(t, t^{\prime}\right)\right)\right.
$$




$$
\begin{aligned}
& \left.+\left(G^{++}\left(t^{\prime}, t\right)-G^{--}\left(t^{\prime}, t\right)-G^{+-}\left(t^{\prime}, t\right)+G^{-+}\left(t^{\prime}, t\right)\right)\right] \\
= & 2 \frac{\partial}{\partial t}\left[G^{>}\left(t, t^{\prime}\right)-G^{<}\left(t, t^{\prime}\right)\right] \theta\left(t-t^{\prime}\right) \\
K\left(t-t^{\prime}\right)= & \frac{1}{4} \frac{\partial^{2}}{\partial t^{2}}\left[G^{++}\left(t, t^{\prime}\right)+G^{--}\left(t, t^{\prime}\right)-G^{+-}\left(t, t^{\prime}\right)-G^{-+}\left(t, t^{\prime}\right)\right] \\
= & \frac{1}{2} \frac{\partial^{2}}{\partial t^{2}}\left[G^{>}\left(t, t^{\prime}\right)+G^{<}\left(t, t^{\prime}\right)\right] .
\end{aligned}
$$

Substituting the values of the Green's functions from eqn.(115) in the above equations, one obtains the expressions for $K_{1}\left(t-t^{\prime}\right)$ and $K\left(t-t^{\prime}\right)$ in eqns. (69) and (70).

In the case that there are bound states other than the zero mode, such as the case of $\phi^{4}$ the sum in eqn.(32) runs over all bound and scattering states, i.e.

$$
\begin{aligned}
F\left[a^{\dagger}, a\right]= & \frac{1}{2 i} \int d p d k \sqrt{\frac{\omega_{p}}{\omega_{k}}} D_{p k}\left[a_{k} a_{p}-a_{-k}^{\dagger} a_{-p}^{\dagger}+a_{-k}^{\dagger} a_{p}-a_{-p}^{\dagger} a_{k}\right] \\
& +\frac{1}{2 i} \sum_{b} \int d k \sqrt{\frac{\omega_{b}}{\omega_{k}}} D_{b k}\left[a_{k} a_{b}-a_{-k}^{\dagger} a_{b}^{\dagger}+a_{-k}^{\dagger} a_{b}-a_{b}^{\dagger} a_{k}\right] \\
& +\frac{1}{2 i} \sum_{b} \int d k \sqrt{\frac{\omega_{k}}{\omega_{b}}} D_{k b}\left[a_{b} a_{k}-a_{b}^{\dagger} a_{-k}^{\dagger}+a_{b}^{\dagger} a_{k}-a_{-k}^{\dagger} a_{b}\right] \\
& +\frac{1}{2 i} \sum_{a, b} \sqrt{\frac{\omega_{a}}{\omega_{b}}} D_{a b}\left[a_{b} a_{a}-a_{b}^{\dagger} a_{a}^{\dagger}+a_{b}^{\dagger} a_{a}-a_{a}^{\dagger} a_{b}\right],
\end{aligned}
$$

where the indices $a$ and $b$ stand for summation over discrete bound states and $p$ and $k$ stand for summation over continuum scattering states. The models which we considered in this paper have at most one bound state, that is the case in the $\phi^{4}$ theory. In this case, the last term will not contribute since $D_{b b}$ vanishes. Thus for only one bound state, eqn.(122) can be written as

$$
\begin{aligned}
F\left[a^{\dagger}, a\right]= & \int d p d k\left[T_{p k}^{(S)}\left(a_{p} a_{k}-a_{-p}^{\dagger} a_{-k}^{\dagger}\right)+T_{p k}^{(A)}\left(a_{-p}^{\dagger} a_{k}-a_{-k}^{\dagger} a_{p}\right)\right] \\
& +\int d k\left[T_{b k}^{(S)}\left(a_{k} a_{b}-a_{-k}^{\dagger} a_{b}^{\dagger}\right)+T_{b k}^{(A)}\left(a_{-k}^{\dagger} a_{b}-a_{b}^{\dagger} a_{k}\right)\right],
\end{aligned}
$$

where the matrices $T_{p k}^{(S)}$ and $T_{p k}^{(A)}$ for scattering states are given by eqn.(33) and if one of the states is a bound state, then

$$
\begin{aligned}
T_{b k}^{(S)} & =\frac{1}{2 i}\left[\sqrt{\frac{\omega_{b}}{\omega_{k}}}-\sqrt{\frac{\omega_{k}}{\omega_{b}}}\right] D_{b k} \\
T_{b k}^{(A)} & =\frac{1}{2 i}\left[\sqrt{\frac{\omega_{b}}{\omega_{k}}}+\sqrt{\frac{\omega_{k}}{\omega_{b}}}\right] D_{b k} .
\end{aligned}
$$

In the Sine-Gordon theory, the last two terms in eqn.(123) do not contribute since in this theory there are no bound states other than the zero mode and the Green's functions are given by eqn.(115) but with integration over $p$ and $k$ instead of the summation. 
In the $\phi^{4}$ case, to lowest adiabatic order the contributions from the bound and scattering states decouple. This implies that the Green's functions will have a contribution from the bound state which is given by the same expression as that of the scattering states, with $p \rightarrow b$, but multiplied by a factor of $1 / 2$ since the bound state wave function is chosen to be real.

\section{GENERALIZED FLUCTUATION-DISSIPATION RELATION}

The functions

$$
\begin{aligned}
G^{>}\left(t-t^{\prime}\right) & =\left\langle F(t) F\left(t^{\prime}\right)\right\rangle \\
G^{<}\left(t-t^{\prime}\right) & =\left\langle F\left(t^{\prime}\right) F(t)\right\rangle,
\end{aligned}
$$

(where $F$ is given by eqn.(32)) admit a spectral representation, and their Fourier transforms in time, $g^{>}(\omega) ; g^{<}(\omega)$ obey the KMS condition 50

$$
g^{<}(\omega)=e^{-\beta \omega} g^{>}(\omega) .
$$

From this expression we find that $k_{1}(\omega)$, the Fourier transform in time of the kernel $i K_{1}\left(t-t^{\prime}\right)=$ $2 \Sigma_{m}\left(t-t^{\prime}\right)$ is given by

$$
k_{1}(\omega)=2 \int \frac{d \omega^{\prime}}{2 \pi} \frac{\omega^{\prime} g^{>}\left(\omega^{\prime}\right)\left[1-e^{-\beta \omega^{\prime}}\right]}{\omega-\omega^{\prime}+i \epsilon},
$$

leading to the imaginary part

$$
\operatorname{Im}\left[k_{1}(\omega)\right]=-\omega g^{>}(\omega)\left[1-e^{-\beta \omega}\right] .
$$

On the other hand the kernel that determines the noise-noise correlation function $K\left(t-t^{\prime}\right)$ has a Fourier transform given by $k(\omega)$ with

$$
\begin{aligned}
k(\omega) & =-\frac{\omega^{2}}{2}\left[g^{>}(\omega)+g^{<}(\omega)\right]=-\frac{\omega^{2}}{2} g^{>}(\omega)\left[1+e^{-\beta \omega}\right] \\
& =\frac{\omega}{2} \operatorname{Im}\left[k_{1}(\omega)\right] \operatorname{coth}\left[\frac{\beta \omega}{2}\right] .
\end{aligned}
$$

The relation between the Fourier transform of the noise-noise correlation function and the imaginary part of the self-energy is the generalized Fluctuation-Dissipation relation[33].

\section{References}

[1] W. P. Su, J. R. Schrieffer and A. J. Heeger, Phys. Rev. Lett. 42, 1698 (1979); Phys. Rev. B 22, 2099 (1980); A. J. Heeger, S. Kivelson, J. R. Schrieffer, and W. P. Su, Rev. Mod. Phys. 60, 781 (1988) and references therein. 
[2] Yu Lu, Kinks and Polarons in Conducting Polymers (World Scientific, Singapore, 1988).

[3] G. Gruner, Density Waves in Solids (Addison-Wesley, New York, 1994); Rev. Mod. Phys. 60, 1129 (1988) and references therein.

[4] V. A. Kuzmin, V. A. Rubakov, and M. E. Shaposhnikov, Phys. Lett. 155B, 36 (1985).

[5] A. G. Cohen, D. B. Kaplan, and A. E. Nelson, Ann. Rev. of Nucl. and Part. Sci. 43, 27 (1993).

[6] E. W. Kolb and M. S. Turner, The Early Universe (Addison Wesley, Redwood City, 1990); A. Linde, Particle Physics and Inflationary Cosmology (Harwood Academic Pub., Switzerland, 1990); R. Brandenberger, Rev. of Mod. Phys. 57, 1 (1985); Int. J. Mod. Phys. A2, 77 (1987).

[7] A. Vilenkin and E. P. S. Shellard, Cosmic Strings and other Topological Defects (Cambridge Monographs on Math. Phys., Cambridge Univ. Press, 1994).

[8] F. J. Alexander, S. Habib, and A. Kovner, Phys. Rev. E 48, 4284 (1993) and references therein.

[9] See for example the summary by Y. Wada, Prog. Theor. Phys. Supp. 113, 1 (1993).

[10] Y. Wada and J. R. Schrieffer, Phys. Rev. B 18, 3897 (1978).

[11] K. Maki, Phys. Rev. B 26, 2181 (1982); 2187;4539; Mol. Cryst. Liq. Cryst. 77, 277 (1981).

[12] M. Ogata and Y. Wada, Prog. Theor. Phys. Supp. 94, 115 (1988); M. Ogata, A. Terai, and Y. Wada, Jour. of the Phys. Soc. of Japan 55, 2305 (1986); ibid. 56, 3220 (1987); M. Ogata and Y. Wada, Jour. of the Phys. Soc. of Japan, 54, 3425 (1985).

[13] A. H. Castro Neto and A. O. Caldeira, Phys. Rev. B 46, 8858 (1992); A. H. Castro Neto and A. O. Caldeira, Phys. Rev. E 48, 4037 (1993); A. H. Castro Neto and A. O. Caldeira, Phys. Rev. A 42, 6884 (1990).

[14] S. Khlebnikov, Phys. Rev. D 46, 3223 (1992).

[15] P. Arnold, Phys. Rev. D 48, 1539 (1993).

[16] R. Rajaraman, Kinks and Instantons An Introduction to Kinks and Instantons in Quantum Field Theory (North-Holland Publishing Co., Amsterdam, 1982).

[17] J. Rubinstein, J. Math. Phys. 11, 258 (1970).

[18] R. Jackiw, Revs. of Mod. Phys. 49 3, 681 (1977); J. Goldstone and R. Jackiw, Phys. Rev. D 11, 1486 (1975); V. E. Korepin, P. P. Kulish, and L. D. Faddeev, JETP lett. 21, 138 (1975).

[19] T. D. Lee and Y. Pang Phys. Rept. 221, 251 (1992).

[20] J. L. Gervais and B. Sakita, Phys. Rev. D 11, 2943 (1975).

[21] J. L. Gervais, A. Jevicki, and B. Sakita, Phys. Rev. D 12, 1038 (1975). 
[22] N. H. Christ and T. D. Lee, Phys. Rev. D 12, 1606 (1975).

[23] E. Tomboulis, Phys. Rev. D 12, 1678 (1975).

[24] M. Creutz, Phys. Rev. D 12, 3126 (1975).

[25] J. L. Gervais and A. Jevicki, Nucl. Phys. B 93, 113 (1976).

[26] H. de Vega, Nucl. Phys. B 115, 411 (1976).

[27] T. D. Holstein and L. Turkevich, Phys. Rev. B 38, 1901 (1988);ibid 1923; T. Holstein, Mol. Crys.Liq. Crys. 77, 235 (1981).

[28] D. Jasnow and J. Rudnick, Phys. Rev. Lett. 41, 698 (1978); J. Rudnick and D. Jasnow, Phys. Rev. B 24, 2760 (1981).

[29] R. Feynman and F. Vernon, Ann. of Phys.(N.Y.) 24, 118 (1963).

[30] A. O. Caldeira and A. J. Leggett, Physica A 121, 587 (1983).

[31] A Schmid, Jour. of Low Temp. Phys. 49, 609 (1982).

[32] H. Grabert, P. Schramm, and G.-L. Ingold, Phys. Rep. 168, 115 (1988).

[33] U. Weiss, Quantum Dissipative Systems (World Scientific, Singapore, 1993) and references therein.

[34] D. Boyanovsky and D.-S. Lee, Nucl. Phys. B[FS] 406, 631 (1993).

[35] J. Schwinger, J. of Math. Phys. 2, 407 (1961).

[36] K. T. Mahanthappa, Phys. Rev. 126, 329 (1962); P. M. Bakshi, and K. T. Mahanthappa, J. Math. Phys. 4, 1, 12 (1963).

[37] L. V. Keldysh, Sov. Phys. JETP 20, 1018 (1965).

[38] V. Korenman, Ann. Phys. 39, 72 (1966).

[39] G. Z. Zhou, Z. B. Su, B. L. Hao, and L. Yu., Phys. Rep. 118, 1 (1985).

[40] J. Rammer and H. Smith, Revs. of Mod. Phys. 58, 323 (1986).

[41] E. M. Lifshitz and L. P. Pitaevskii, Physical Kinetics (Pergamon, N.Y., 1981); G. D. Mahan, Many Particle Physics, 2nd ed (Plenum, N.Y., 1990); H. Kleinert, Path Integrals in Quantum Mechanics, Statistics and Polymer Physics, 2nd ed (World Scientific, Singapore, 1996); R. Mills, Propagators for Many Particle Systems (Gordon and Breach, N.Y., 1969).

[42] L. P. Kadanoff and G. Baym, Quantum Statistical Mechanics (Benjamin, N.Y., 1962). 
[43] E. Calzetta and B. L. Hu, Phys. Rev. D 35, 495 (1987); Phys. Rev. D 37, 2878 (1988); Phys. Rev. D 40, 656 (1989) and references therein.

[44] J. W. Negele and H. Orland, Quantum Many-Particle Systems (Addison-Wesley, 1988).

[45] P. Morse and H. Feshbach, Methods of Mathematical Physics (McGraw-Hill, 1953) p. 1650.

[46] D. Boyanovsky, M. D’Attanasio, H.J. de Vega, and R. Holman, Phys. Rev. D 54, 1748 (1996).

[47] H. A. Weldon, Phys. Rev. D 28, 2007 (1983).

[48] E. Braaten and R. D. Pisarski, Nucl. Phys. B 337, 569 (1990); E. Braaten and R. D. Pisarski, Nucl. Phys. B 339, 310 (1990); E. Braaten and R. D. Pisarski, Phys. Rev. Lett. 64, 1338 (1990); R. D. Pisarski, Phys. Rev. Lett. 63, 1129 (1989); E. Braaten and R. D. Pisarski, Phys. Rev. D 42, 2156 (1990).

[49] D. Boyanovsky, F. Cooper, H. J. de Vega and P. Sodano, to appear in Phys. Rev. D (hep$\mathrm{ph} / 9802277$.

[50] A. L. Fetter and J. D. Walecka, Quantum Theory of Many Particle Systems (McGraw-Hill, San Francisco, 1971), (Chapter 9). 


\section{Figure Captions}

Figure 1 The non-equilibrium one-loop contributions to the self energy. The upper two contributions correspond to emission-annihilation of two mesons. The lower two correspond scattering off in-medium mesons and responsible for Landau damping.

Figure 2 Contour in the complex s-plane for the inverse Laplace transform.

Figure 3 The functions $\Gamma(\tau)$ and $\Sigma(\tau)$ for temperatures $\mathcal{T}=0,1.0,5.0$ and 10.0 for SineGordon theory.

Figure 4 Numerical evaluation of the velocity of the kink for $j=0 ; v_{0}=1$ for temperatures $\mathcal{T}=0,1.0,5.0$ and 10.0 in Sine-Gordon theory.

Figure 5 The correlation function $\mathcal{K}(\tau)$ for temperatures $\mathcal{T}=0,1.0,5.0$ and 10.0 in the Sine-Gordon theory.

Figure 6 The functions $\Gamma(\tau)$ and $\Sigma(\tau)$ for temperatures $\mathcal{T}=0,1.0,5.0$ and 10.0 in the $\phi^{4}$-theory. Contributions from bound and scattering states are displayed separately.

Figure 7 Numerical evaluation of the velocity of the kink for $j=0 ; v_{0}=1$ for temperatures $\mathcal{T}=0,1.0,5.0$ and 10.0 in $\phi^{4}$ theory.

Figure 8 The correlation function $\mathcal{K}(\tau)$ for temperatures $\mathcal{T}=0,1.0,5.0$ and 10.0 in the $\phi^{4}$ theory.

Figure 9 Integrals $I_{1} ; I_{2}$ corresponding to the contributions from $\Sigma_{1} ; \Sigma_{2}$ to the self-energy kernel at $\tau=0$ vs. $\mathcal{T}$ in the Sine Gordon theory. 


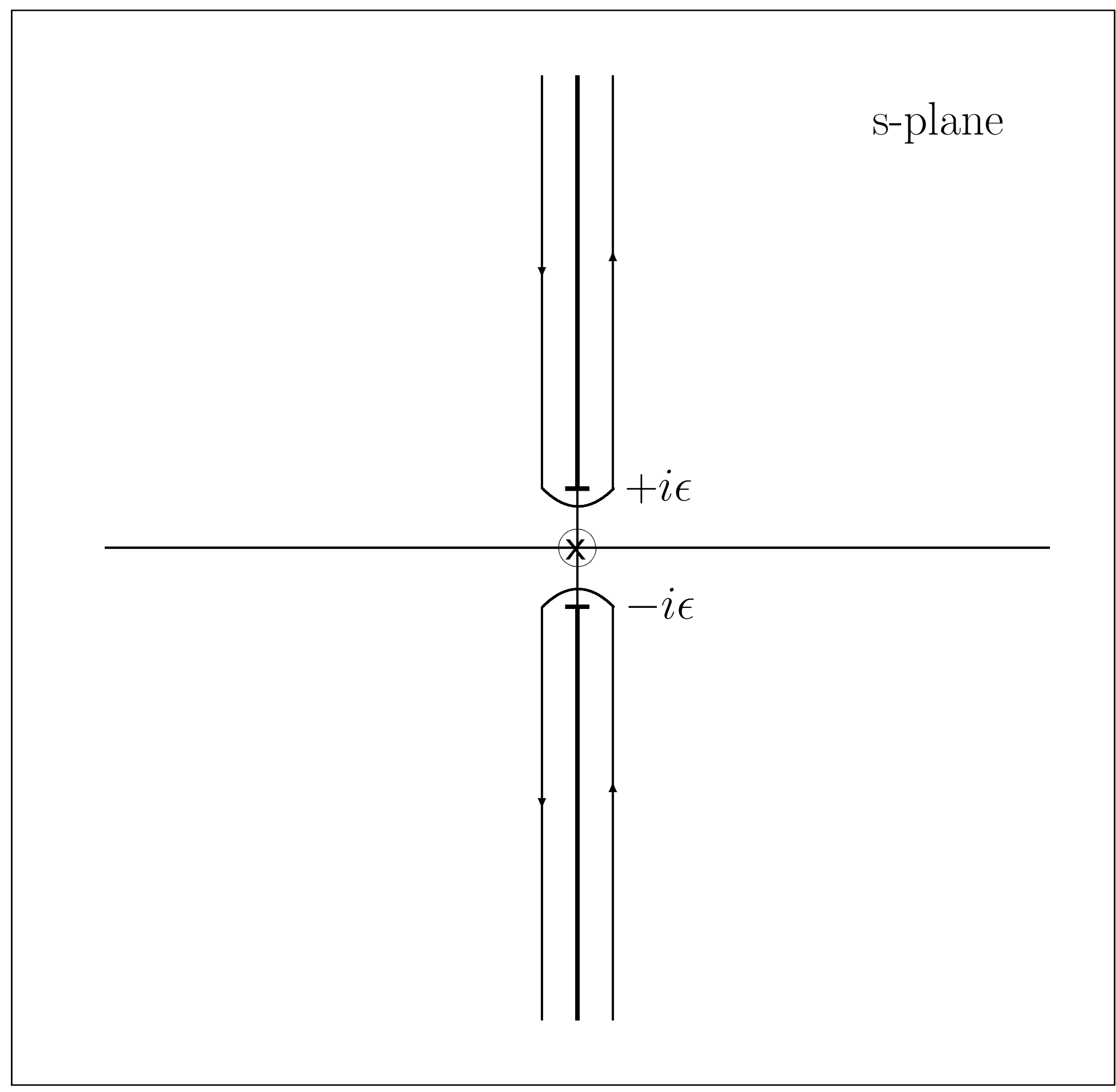


This figure "fig3.gif" is available in "gif" format from: http://arxiv.org/ps/hep-ph/9710359v2 
This figure "fig4.gif" is available in "gif" format from: http://arxiv.org/ps/hep-ph/9710359v2 
This figure "fig5.gif" is available in "gif" format from: http://arxiv.org/ps/hep-ph/9710359v2 
This figure "fig6.gif" is available in "gif" format from: http://arxiv.org/ps/hep-ph/9710359v2 
This figure "fig7.gif" is available in "gif" format from: http://arxiv.org/ps/hep-ph/9710359v2 
This figure "fig8.gif" is available in "gif" format from: http://arxiv.org/ps/hep-ph/9710359v2 
